\title{
I LUOGHI DELLA MEMORIA FUNERARIA: RIFLESSIONI SU FORME E CONTESTI DELLE EPIGRAFI SEPOLCRALI DI AMBITO MONASTICO (ETÂ LONGOBARDA E CAROLINGIA)
}

\section{DANIELE FERRAIUOLO}

\author{
UDC: $27-788 " 653^{\prime \prime}$ \\ 27-55"653" \\ Preliminary communication \\ Manuscript received: 28. 10. 2016. \\ Revised manuscript accepted: 07. 02. 2017. \\ DOI: 10.1484/J.HAM.5.113748
}

\author{
D. Ferraiuolo \\ Università degli Studi Suor Orsola Benincasa di Napoli \\ LATEM (Laboratorio di Archeologia Tardoantica e Medievale) \\ Via Sorgente, 4 \\ 81016 Piedimonte Matese (Ce), Italia
}

Starting from italian, swiss and french documentation in lombard and carolingian age, the study examines the relationships between inscriptions and the space in which they themselves are inserted. This paper, concerning specifically the monastic context, proposes various hypotheses on burial signaling mode, taking into account the physical appearance of epigraphs, as well as their degree of visibility in relation to its surroundings. Through a comparative analysis, which also involves the main historical sources of the time, it is reflected on the location of the tombs of monks, abbots and laity within the monastic space, thus arriving at a number of proposals on the original location of the epigraphic artifacts.

Keywords: medieval epigraphy; medieval monastic life; funerary inscriptions; funerary practices.

Tra i precetti principali che regolano l'organizzazione cenobitica in età altomedievale, il rapporto di stabilità che i monaci sono tenuti a rispettare nei confronti della propria familia mostra di oltrepassare i semplici limiti delle attività quotidiane, coinvolgendo anche la sfera puramente funeraria. Le parole di Isidoro di Siviglia contenute nella Regula monachorum mostrano la necessità che i monaci siano sepolti tutti insieme in un unico luogo, al fine di tenere unito lo spirito di carità dinanzi ai monaci viventi ${ }^{1}$. Gli insegnamenti del vescovo spagnolo mostrano, in realtà, un loro attecchimento in Italia a partire dall'VIII secolo, momento cui le indagini archeologiche assegnano la comparsa di cimiteri collettivi topograficamente in linea con le chiese abbaziali, contrassegnati da tombe allineate di varia tipologia a seconda dell'insediamento monastico. E qui si potrà richiamare il caso emblematico di San Salvatore di Brescia, dove le indagini condotte da Gian Pietro Brogiolo hanno evidenziato la presenza di due aree funerarie ben distinte, di cui la prima, posta a sud della chiesa abbaziale di VIII secolo, ha restituito un numero ingente di tombe che si aggira intorno alle cento unità ${ }^{2}$; diversamente, le aree poste a ridosso della facciata e in corrispondenza della navata sembrano essere state destinate, invece, alla sepoltura delle badesse, come lascia immaginare la presenza di tombe dipinte di tipo privilegiato3.

Una simile pianificazione topografica sembra aver interessato, senza sostanziali distinzioni, anche i monasteri

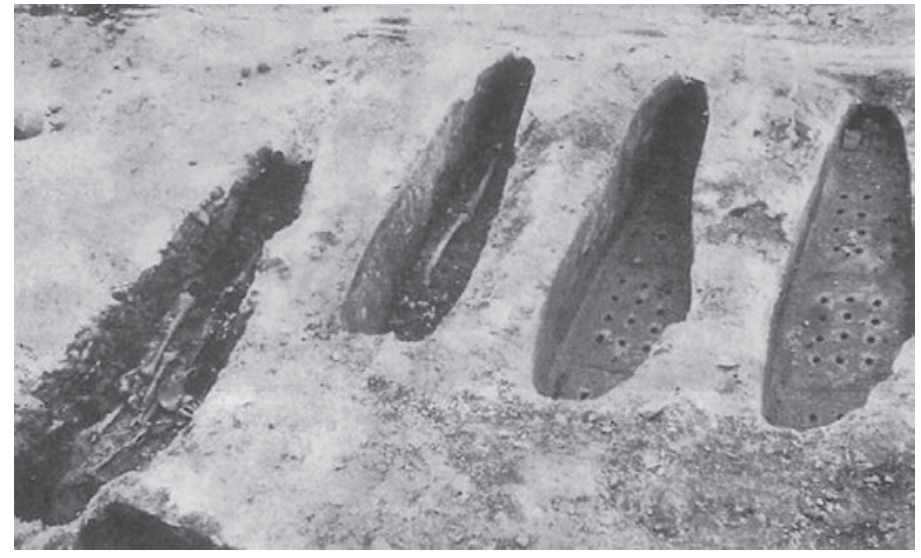

Fig. 1. Alcune sepolture rinvenute nel cimitero monastico indagato da Angelo Pantoni, assegnabili alla fase altomedievale del cenobio di Montecassino (da A. PANTONI, 1953).

ubicati nelle zone centro-meridionali della penisola. A Montecassino, ad esempio, le indagini eseguite da Angelo Pantoni in corrispondenza dell'area occupata in origine dalla biblioteca e dalle cucine hanno evidenziato la presenza di un'area cimiteriale aperta destinata ai monaci, di cui sono state indagate circa ottanta sepolture (fig. 1); analogamente, il monaco cassinese ipotizzava l'esistenza di spazi ben distinti per le sepolture privilegiate, individuando nel portico della chiesa di San Martino, ovvero nella chiesa di San Giovanni, i luoghi più consoni a questa particolare destinazione d'uso ${ }^{4}$.

${ }^{1}$ Patrologia latina, ed. J.P. Migne, 83, col. 894: «corpora fratrum uno sepelienda sunt loco, ut quos viventes charitatis tenuit unitas, morientes unus locus amplectatur». Su questo argomento si veda, in particolare, il paragrafo intitolato "Le cimetière des moines, modèle d'un cimetière communautaire", in C. TREFFORT, L’Église carolingienne et la mort. Christianisme, rites funéraires et pratiques commémoratives, Lyon, 1996, p. 154.

${ }^{2}$ G.P. BROGIOLO, Sepolture privilegiate altomedievali nel monastero di S. Salvatore di Brescia, in Hortus artium medievalium, 10, 2004, p. 15-24.

3 Il gruppo delle sepolture della navata fu individuato da Gaetano Panazza nel 1957-1962, per cui si veda G. PANAZZA, Gli scavi, l'architettura e gli affreschi della chiesa di San Salvatore in Brescia, in G. Panazza, A. Peroni (dir.), La chiesa di San Salvatore in Brescia, Atti dell'VIII Congresso di Studi sull'Arte dell'Alto Medioevo, II, Milano, 1962, p. 363-391. Sulle sepolture dipinte, S. STRAFELLA, Sepolture dipinte nel monastero di San Salvatore, in G.P. Brogiolo, F. Morandini (dir.), Dalla corte regia al monastero di San Salvatore-Santa Giulia di Brescia, Verona, 2014, p. 255-267.

${ }^{4}$ A. PANTONI, Su di un cimitero altomedievale a Montecassino e sul sepolcro di Paolo Diacono, in Atti del $2^{\circ}$ Congresso Internazionale di Studi sull'Alto Medioevo (Grado-Aquileia-Gorizia-Cividale-Udine, 7-11 settembre 1952), Spoleto, 1953, p. 257-264. 
Pur nei limiti imposti dai principi della "stabilità" e dal sentimento di aggregazione che contraddistingue le comunità, la distribuzione delle aree funerarie in ambito monastico non può non aver tenuto conto del fattore gerarchico, mostrando una ripartizione degli spazi avvenuta quasi sempre in funzione del livello sociale del defunto. I casi europei archeologicamente documentati mostrano una predilezione per le aree esterne delle chiese per l'inumazione dei monaci, mentre per gli abati e i personaggi illustri mostra di prevalere il modello che vede l'uso di spazi interni e dell'ingresso degli edifici di culto per la loro sepoltura 5 . Ciò nonostante, lo status sociale mostra, talvolta, di condizionare fortemente la scelta del luogo di inumazione, indicando in alcuni casi addirittura l'invasione di spazi riservati a diverse categorie. È il caso del monastero femminile di Hamage, nel nord della Francia, dove le indagini archeologiche hanno evidenziato la presenza di sepolture di VIII secolo riconducibili ad un unico gruppo familiare aristocratico composto anche da monache ${ }^{6}$. In questo esempio, l'eccezione alla regola sembra essere costituita dall'atto stesso della sepoltura e dall'occupazione di un luogo "altro" rispetto agli spazi appena citati.

Più complessa ed articolata è, invece, la vicenda che vede la proliferazione di chiese monastiche a destinazione propriamente funeraria. A partire dall'età carolingia, sembra farsi maggiormente strada l'idea di ospitare all'interno di chiese isolate consacrate $a d$ hoc, o nelle loro immediate vicinanze, i membri della comunità, includendo in alcuni casi anche gli abati ${ }^{7}$. Nell'819, l'abate di Fulda Eigil (818-822) avviò di sua iniziativa l'edificazione di una chiesa dedicata a San Michele, a completamento di un imponente progetto edilizio che aveva visto impegnato in prima battuta il suo predecessore, l'abate Ratgar (802-817). La Vita Eigilis abbatis fuldensis ricorda, infatti, la costruzione di una basilica parva, «ubi defuncta corpora fratrum sepulturae tradita requiescunt, quam cimiterium vocant $»^{8}$, documentando in questo modo una destinazione d'uso dell'edificio avvenuta già a partire dal suo concepimento. In realtà, le parole del biografo non lasciano dubbi anche sul fatto che l'edificio andò ad occupare un'area funeraria già esistente, un cimiterium per l'appunto, allo scopo di supportare ulteriormente le anime dei fratelli defunti e porvi così le spoglie dell'abate ${ }^{9}$.
Evitando di incorrere in eccessive argomentazioni che oltrepassino i limiti preposti dal presente contributo, un aspetto su cui risulterà utile avanzare alcune riflessioni è quello relativo, nello specifico, alle modalità di segnalazione delle singole sepolture. In quest'ambito, dove è possibile attestare variazioni che scaturiscono da caso a caso, anche la scrittura esposta assume il ruolo di connettore tra il mondo dei vivi e quello dei morti, attraverso forme e contenuti che rispondono alle necessità dettate da ogni singola comunità monastica e dagli spazi destinati, come si avrà modo di vedere, alla loro commemorazione.

Chi intenda affrontare l'aspetto riguardante il contesto d'origine delle epigrafi, deve necessariamente misurarsi con l'estrema rarità dei manufatti superstiti conservati ancora in situ. La quasi totalità dei prodotti sottoposti ad analisi è, infatti, fuori contesto, ragion per cui si è ritenuto indispensabile intraprendere la ricerca partendo da una rapida distinzione tipologica dei manufatti documentabili in campo monastico, tenendo conto della sola produzione lapidea.

Una suddivisione maturata in ambito francese - concernente la sfera propriamente funeraria di età carolingia - ha già proposto l'esistenza di tre macro-raggruppamenti epigrafici, sulla base dei rapporti intercorrenti tra iscrizione e spazio funerario di riferimento ${ }^{10}$. L'esame parte dalle plates tombes, il cui corrispettivo italiano è quello delle lapidi tombali. Si tratta di lastre più o meno spesse e di dimensioni variabili ubicate a copertura di una tomba terragna o a cassa, poste prevalentemente in spazi chiusi, benché non manchino esemplari documentati in aree aperte ${ }^{\text {I. }}$. Segue la categoria delle stele funerarie poste in posizione verticale in corrispondenza della sepoltura e di dimensioni variabili a seconda del messaggio riportato: in questo caso, si prevede una collocazione in ambiente aperto e in rapporto a fosse terragne ${ }^{12}$. Composto da tipologie epigrafiche eterogenee, l'ultimo insieme determinato, quello delle inscriptions pariétales, comprende, invece, al proprio interno epigrafi dipinte o eseguite su supporto mobile ${ }^{13}$. In quest'ultimo caso, l'iscrizione ha sempre rapporto indiretto con il defunto, mentre diretta è la relazione con l'osservatore, per il fatto di essere prevalentemente ubicata dinanzi agli occhi del lettore stesso, ovvero in posizione tale da poterne consentire l'osservazione.

${ }_{5}^{5}$ Il dato archeologico documenta anche alcuni casi "eccezionali" riguardanti le sepolture di abati e badesse. Al di là dei casi inerenti alle pratiche di tumulazione all'interno della chiesa abbaziale o in prossimità del suo ingresso, le ricerche archeologiche testimoniano l'utilizzo di altri spazi, quali il cimitero

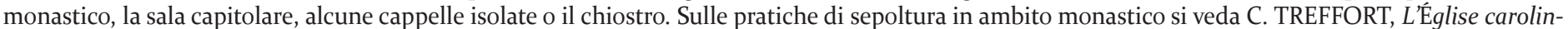
gienne et la mort, op. cit. (n. 1); inoltre, da ultimo, corredato da alcuni interessanti esempi, il contributo di G. CANTINO WATAGHIN, E. DESTEFANIS, Les espaces funéraires dans les ensembles monastiques du haut moyen age, in M. Lauwers (dir.), Monastères et espace social. Genèse et transformation d'un système de lieux dans l'occident médiéval, Turnhout, 2014, p. 503-553, in part. p. 512-524; sulle sepolture degli abati, Ibidem, p. 525-532.

${ }^{6}$ É. LOUIS, Hamage (Nord). Ėspaces et bâtiments d'un monastère mérovingien et carolingien, in P. Racine (dir.), Pratique et sacré dans les éspaces monastiques au Moyen-Âge et à l'époque moderne, Amiens, 1997, p. 73-97; IDEM, Sorores er fratres in Hamatico degentes. Naissance, évolution et disparition d'une abbaye au Haut Moyen-Âge: Hamage (France, Nord), in Les monastères mérovingiens et leur domaines, Actes du colloque de Logne, 26 septembre 1998, in De La Meuse à l'Ardenne, 29, 1999, p. 15-47.

7 Si tratta di un fenomeno osservabile già nel VII secolo in ambito francese, così come testimoniato dai casi di Nivelles o Sithiu (Ibidem, p. 523).

${ }^{8}$ Candidus, Sancti Eigilis abbatis Fuldensis vita auctore Candido monacho Fuldensi, in Monumenta Germaniae Historica (= MGH), Scriptores rerum Langobardicarum et Italicarum, ed. G. Waitz, Hannoverae 1887, XV, 1, p. 222-233, in part. p. 230.

${ }_{9}^{9}$ Sull'attivita di Eigil a Fulda si veda J. RAAIJMAKERS, The making of the monastic community of Fulda, c. 744-c. 90o, Cambridge, 2012, p. 132-174. Su questo aspetto in particolare, riguardante l'occupazione dell'area funeraria monastica, cfr. Ibidem, p. 133. Si veda inoltre, per la chiesa di San Michele, O. ELLGER, Die Michaelskirche zu Fulda als Zeugnis der Totensorge. Zur Konzeprion einer Friedhofs und Grabkirche im karolingischen Kloster Fulda, Fulda, 1989 (Veröffentlichung des Fuldaer Geschichtsvereins, 55).

${ }^{10}$ C. TREFFORT, Mémoires carolingiennes. L'épitaphe entre célébration mémorielle, genre littéraire et manifeste politique (milieu VIII ${ }^{e}$-début XI ${ }^{e}$ siècle), Rennes, 2007, p. 120-130.

"Ibidem, p. 121-122.

${ }^{12}$ Ibidem, p. 122-123.

${ }_{13}$ Ibidem, p. 127-129. 
A partire da questi esempi, sarà utile ricordare un recente esame riguardante i modelli impaginativi delle iscrizioni elitarie longobarde e carolinge ${ }^{14}$, che ha posto l'attenzione sui rapporti di interdipendenza che le iscrizioni mostrano di instaurare con lo spazio al cui interno esse stesse sono inserite. Tutto ciò presuppone che a modifiche inerenti le pratiche funerarie dovettero corrispondere, specie nel passaggio dall'età longobarda a quella carolingia, variazioni riguardanti le forme delle epigrafi e l'aspetto inerente alla mise en page. Ė stato dimostrato, infatti, come il passaggio da lastre a sviluppo verticale a impianti di tipo orizzontale costituisca, in effetti, il prodotto di alcune variazioni che videro una graduale sostituzione delle tombe terragne da parte di sepolture isolate ad arcosolio e di tipo monumentale ${ }^{15}$.

Se per la produzione epigrafica di ambito laico ed elitario la distinzione mostra di essere circoscritta a tipologie, per così dire, "canoniche", diverso è il quadro delle scritture epigrafiche ad uso e consumo dei monasteri. La complessità morfologica del campione ha comportato, in questo caso, una ripartizione che predilige l'aspetto dimensionale come parametro modello, permettendo in questo modo

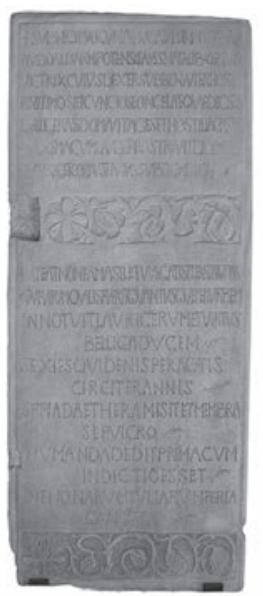

$2 \mathrm{a}$

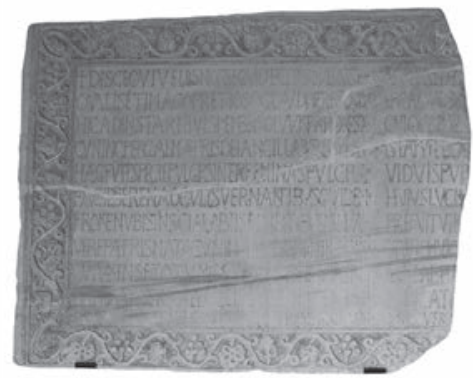

$2 \mathrm{e}$

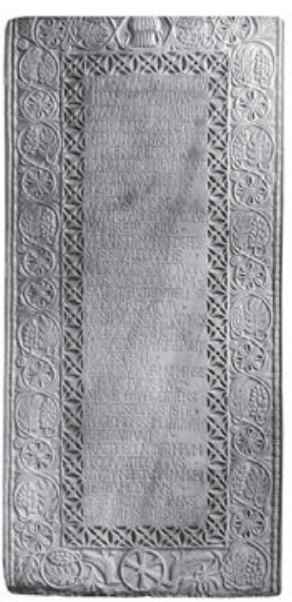

$2 b$

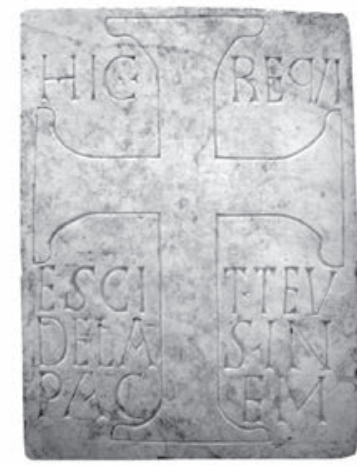

$2 c$

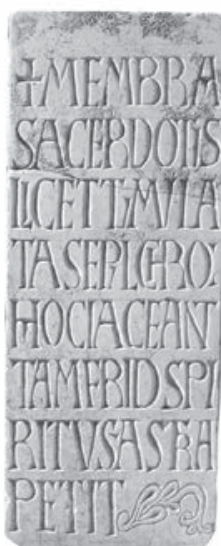

$2 \mathrm{~d}$

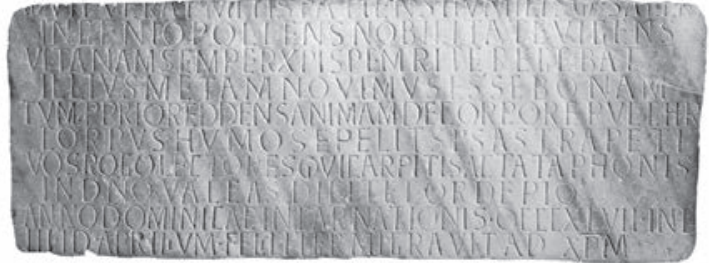

$2 \mathrm{f}$

Fig. 2. Schema delle principali tipologie epigrafiche funerarie di ambito italiano (grandi dimensioni, secoli VIII-IX).

e secolare ha registrato l'esistenza di iscrizioni rettangolari di grandi dimensioni, connotate dalla presenza di un testo sviluppato parallelamente al lato breve del manufatto (sviluppo verticale) o, al contrario, al suo lato lungo (sviluppo orizzontale) (fig. 2).

Iniziando il discorso a partire dalle epigrafi con sviluppo verticale (figg. 2a-2d), il loro utilizzo in ambito monastico per tutto l'VIII e il IX secolo non sembra essere connesso in nessun caso alla memoria dei monaci, mostrando, invece, di essere riservato agli abati e ad alcuni personaggi dell'entourage ecclesiastico. Appartengono a questa tipologia, per citare gli esempi maggiormente rappresentativi, le iscrizioni dell'abate Cumiano ${ }^{16}$ (fig. 2b) e di un secondo ignoto da Bobbio ${ }^{17}$, della badessa Ermingarda ${ }^{18}$ di San

Una stima eseguita sul campione di epigrafi destinate ad abati/badesse ed esponenti della gerarchia ecclesiastica

\footnotetext{
${ }^{14}$ F. DE RUBEIS, Modelli impaginativi delle iscrizioni funerarie elitarie tra Longobardi e Carolingi, in Scripta. An international journal of codicology and palaeography, 6, 2013, p. 57-66.

${ }^{15}$ Su questo aspetto in particolare, si vedano le ipotesi riportate Ibidem, p. 62-65.

${ }^{16}$ Per l'epigrafe di Cumiano (Bobbio, Museo dell'Abbazia), si veda A. SILVAGNI, Monumenta Epigraphica Christiana saeculo XIII antiquiora quae in Italiae finibus adhuc extant, III. Papia, Città del Vaticano, 1943, tab. IV, $\mathrm{n}^{\circ}$ 8; N. GRAY, The palaeography of latin inscriptions in the Eight, Ninth and Tenth centuries in Italy, in Papers of British School at Rome, XVI, new series, III, 1948, p. 38-163, in part. p. 70, n ${ }^{\circ} 33$; A. SEGAGNI MALACART, La scultura in pietra dal VI al X secolo, in Storia di Pavia, II, Milano, 1987, p. 374-406, in part. p. 382-383; A. PETRUCCI, Le scritture ultime. Ideologie della morte e strategie dello scrivere nella tradizione occidentale, Torino, 1995, p. 54; S. LOMARTIRE, L'iscrizione di Cumiano e l'epigrafia longobarda dell'età liutprandea, in F.G. Nuvolone (dir.), La fondazione di Bobbio nello sviluppo delle comunicazioni tra Langobardia e Toscana nel medioevo, Atti del convegno internazionale (Bobbio, 1-2 ottobre 1999), Piacenza-Bobbio, 2000, p. 57-70; E. DESTEFANIS, Materiali lapidei e fittili di età altomedievale da Bobbio, Piacenza, 2004, p. 108, n 9 ; EAD., Corpus della scultura altomedievale, XVIII. La diocesi di Piacenza e il monastero di Bobbio, Spoleto, 2008, p. 74-75, $n^{\circ} 102$; G. CANTINO WATAGHIN, Cantieri monastici nell'alto medioevo in Italia settentrionale, in Cantieri e maestranze nell'Italia medievale, Atti del convegno di studi (Chieti-San Salvo, 16-18 maggio 2008), Spoleto, 2010 (De re monastica, II), p. 279-344, in part. p. 323-325.

${ }^{17}$ Si vedano le schede in dettaglio, a cura di E. DESTEFANIS, Materiali lapidei e fittili, op. cit. (n. 16), p. 108, n. 9; EAD., Corpus della scultura altomedievale, op. cit. (n. 16), p. 121-128, p. 10-13. Per ulteriori studi si vedano S. LOMARTIRE, L'iscrizione di Cumiano, op. cit. (n. 16), p. 68; A. CALZONA, Bobbio, frammento di lastra con iscrizione, riutilizzata come mensola, in A.C. Quintavalle (a cura di), Il medioevo delle cattedrali. Chiesa e impero: la lotta delle immagini (secoli XI e XII), Milano, 2006, p. 447-450, n. 18.

${ }^{18}$ Cfr. F. DE RUBEIS, Le iscrizioni dei re longobardi, in F. Stella (a cura di), Poesia dell'alto medioevo europeo: manoscritti, lingua e musica dei ritmi latini, Atti delle Euroconferenze per il Corpus dei ritmi latini, IV-IX sec. (Arezzo, 6-7 novembre 1998; Ravello, 9-12 settembre 1999), Firenze, 2000, p. 223-237, in part. p. 236.
} 


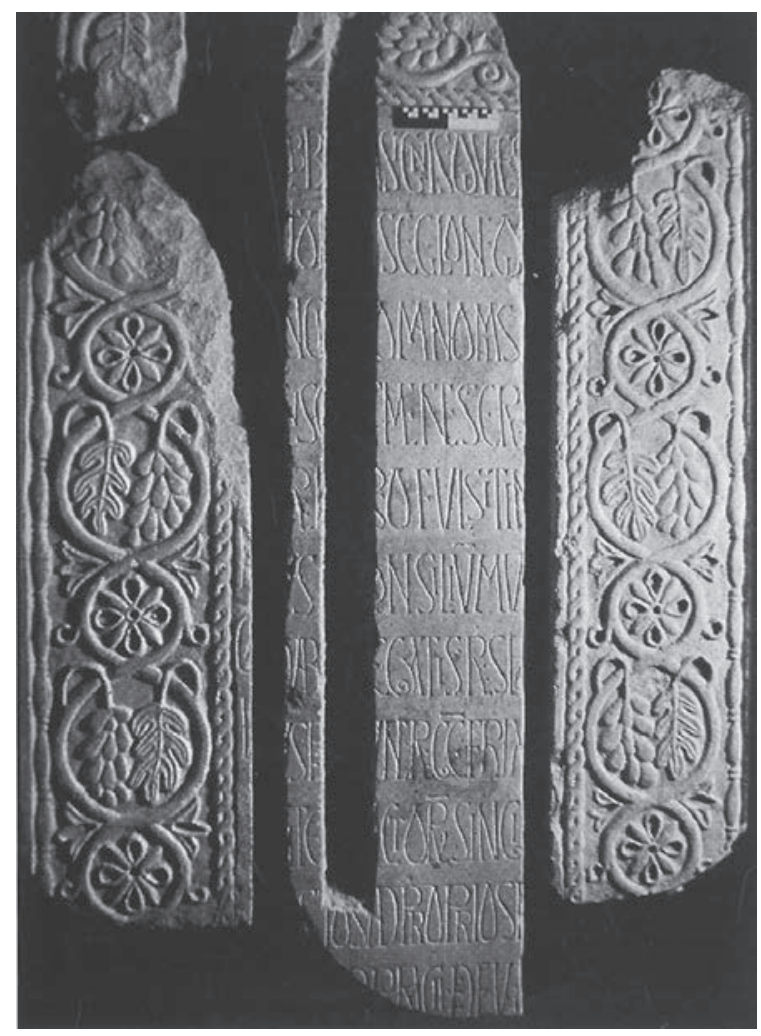

Fig. 3. Bobbio, Museo dell'Abbazia, epigrafe di personaggio ignoto (prima metà VIII secolo, da E. DESTEFANIS, 2002).

Salvatore di Brescia, di Anselmus da Leno ${ }^{19}$, del sacerdote Tamfrid $^{20}$ (fig. 2d) e di un non meglio identificato Teudelas da San Vincenzo al Volturno (fig. 2c). Nella maggioranza dei casi, l'analisi del testo non lascia dubbi sul rapporto di contiguità fisica dell'iscrizione con il sepolcro, mostrando l'utilizzo di espressioni del tutto indicative in questo senso, quali «ubi tegitur corpus» e «tymbum» $»^{21}$, «manomissione» $»^{22}$, «tumulata sepulchro»³.

Bisognerà tentare di immaginare una sistemazione delle lastre all'interno di un contesto quasi sicuramente chiuso, trattandosi di messaggi rivolti prevalentemente alla memoria degli abati. Le opposte direzioni di lettura del tralcio nella lastra di Cumiano hanno suggerito, ad esempio, una collocazione terragna dell'oggetto in corrispondenza di uno spazio abbastanza ampio da permetterne l'osservazione da diverse angolazioni, fattore che ha spinto, in seconda battuta, a suggerire l'ubicazione della tomba in un eventuale portico o sacello indipendente ${ }^{24}$. L'assenza di tracce che riconducano in qualche modo all'usura per calpestio, ha contribuito, inoltre, a far nascere l'idea che il sepolcro, e così l'epigrafe, fossero

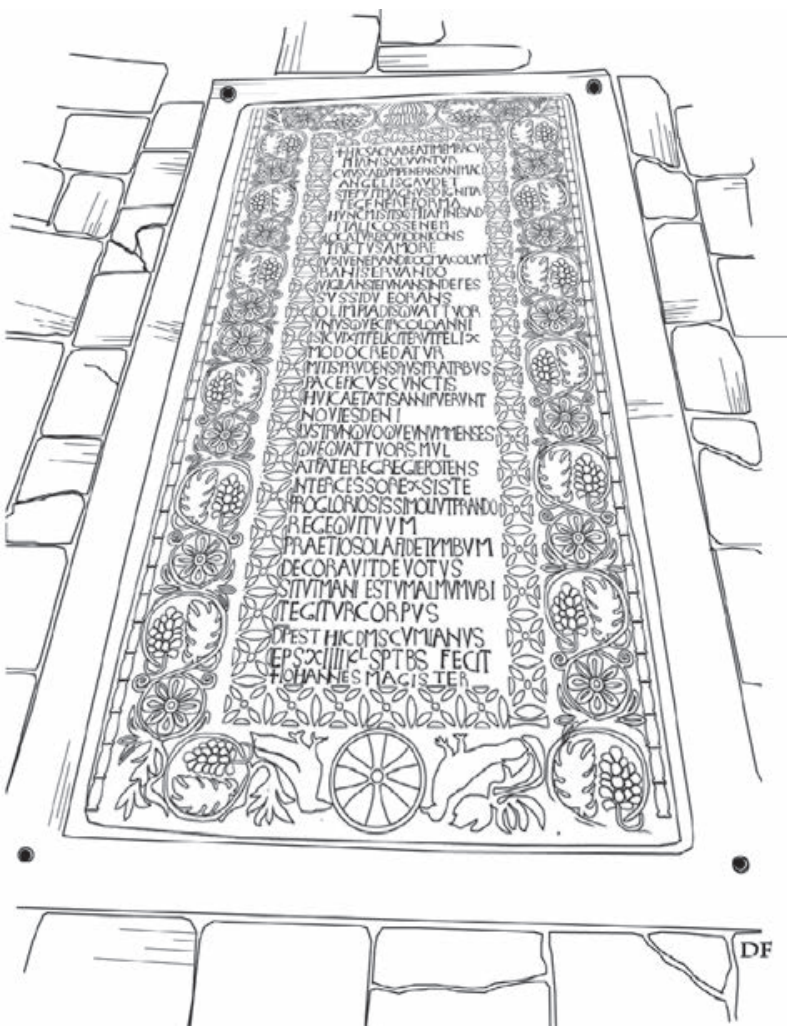

Fig. 4. Restituzione ideale di tomba terragna con lastra epigrafica di copertura (es. epigrafe di Cumiano da Bobbio).

protetti da una sorta di recinzione transennata ${ }^{25}$, assegnando così alla lastra tombale prerogative di carattere monumentale e enfatizzandone il valore attraverso la scrittura.

Rientra nelle finalità del medesimo progetto di monumentalizzazione, infatti, anche l'epigrafe di un ignoto abate (fig. 3), ubicata verosimilmente nello stesso luogo, così che, volendo parafrasare le parole di Eleonora Destefanis, «il complesso cultuale diviene, in una pluralità di significati, uno "spazio della memoria" o "memoria fatta spazio", nel quadro di una politica di promozione di chiese e monasteri perseguita da Liutprando ${ }^{26}$. Le ipotesi che vedono la collocazione delle lastre a pavimento possono reggersi anche sull'aspetto dimensionale dei manufatti, mostrando, ad esempio, per l'iscrizione di Cumiano una lunghezza di 179 $\mathrm{cm}$ e una larghezza di $90 \mathrm{~cm}$, corrispondente, grosso modo, all'ampiezza di una comune tomba a cassa (fig. 4).

Passando a considerare gli esemplari al cui interno il testo risulta orientato parallelamente al lato lungo della lastra, bisognerà partire da una distinzione operata in ambito elitario. Durante tutto l'VIII secolo, le aree settentrionali

\footnotetext{
${ }^{19}$ M. SANNAZARO, Le iscrizioni paleocristiane e altomedievali da Leno. Alcune osservazioni, in Brixia sacra. Memorie storiche della diocesi di Brescia, III ser., XI, 2, 2006, p. 339-352, in part. p. 346-347.

${ }^{20}$ Sull'epigrafe (San Vincenzo al Volturno, RN 2876), si veda F. DE RUBEIS, La scrittura a San Vincenzo al Volturno fra manoscritti ed epigrafi, in F. Marazzi (a cura di), San Vincenzo al Volturno. Cultura, istituzioni, economia, Monteroduni (Is), 1996, p. 21-40, in part. p. 31; J. MITCHELL, Late antique and early medieval carved inscriptions, in J. Mitchell, I.L. Hansen (dir.), San Vincenzo al Volturno 3: the finds from the 1980-86 excavations, Text and Illustrations, Spoleto, 2001, p. 33-82, in part. p. 50; D. FERRAIUOLO, Spolien und Exponate aus San Vincenzo al Volturno: Inschriften, in Macht des Wortes. Benediktinisches mönchtum im spiegel Europas, II. Katalog, Regensburg, 2009, p. 113-115, in part. p. 114; ID., Scrivere, impaginare, scolpire. L'officina lapidaria a San Vincenzo al Volturno (secc. VIII-IX), in F. Marazzi (a cura di), Archeologia della parola. Percorsi e strumenti per la tradizione della memoria nel monastero di San Vincenzo al Voltuno, Morcone (Bn), 2009, p. 79-100, in part. p. 92-93; ID., Tra canone e innovazione. Lavorazione delle epigrafi nella Langobardia Minor (secoli VIII-X), Firenze, 2013, p. 83-84, n. 4.

${ }^{21}$ Per l'epigrafe di Cumiano.

${ }_{22}^{22}$ Visibile nell'epigrafe dell'ignoto abate di Bobbio.

${ }^{23}$ In corrispondenza della terza e della quarta riga dell'iscrizione di Tamfrid, da San Vincenzo al Volturno.

${ }^{24}$ E. DESTEFANIS, Il monastero di Bobbio in età altomedievale, Firenze, 2002, p. 41.

${ }^{25}$ Ibidem, p. 42.

${ }^{26}$ Ibidem, p. 43-44.
} 


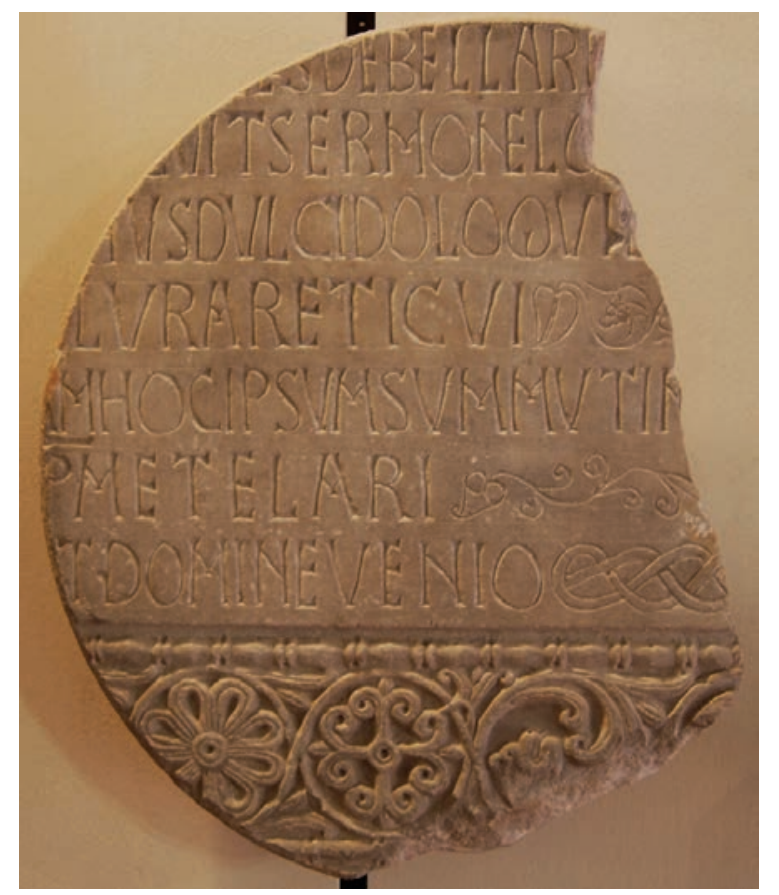

Fig. 5. Pavia, Musei Civici del Castello Visconteo. Frammento di epigrafe di badessa (?) (metà VIII secolo).

della penisola mostrano l'adesione ad un modello epigrafico funerario di tipo riservato, con impianto di impaginazione orizzontale e testo disposto su doppia colonna. Questo tipo di soluzione sembra rispondere, oltre che ad esigenze metriche dettate dall'articolazione dei testi, anche ad un fenomeno di "monumentalizzazione" delle sepolture regie. In ambito monastico, questo tipo di produzione mostra di essere circoscritto solo ad alcuni casi eccezionali, tutti inquadrabili nell'ambito delle manifestazioni pavesi di corte. Epigrafi a doppia colonna e di grandi dimensioni sono di fatti destinate alla badessa Cuniperga ${ }^{27}(200 \times 88 \mathrm{~cm}$, cfr. fig. 2e), alle badesse del monastero di Sant'Agata al Monte di Pavia e alla badessa Teodota ${ }^{28}$ dell'omonimo cenobio pavese $(200 \times 95 \mathrm{~cm})$. In tutti i casi, il testo in versi è distribuito su due colonne parallele, di 11 versi per l'epigrafe di Cuniperga e di 15 per quella di Teodota, mentre per il gruppo di "Casa Anelli", concernente il monastero di Sant'Agata al Monte ${ }^{29}$, si può solo presumere una suddivisione in colonne, senza tuttavia risalire al numero dei versi in esse contenuti (fig. 5).

Tra le diverse ipotesi, le più recenti interpretazioni hanno proposto una sistemazione a parete di questa par-

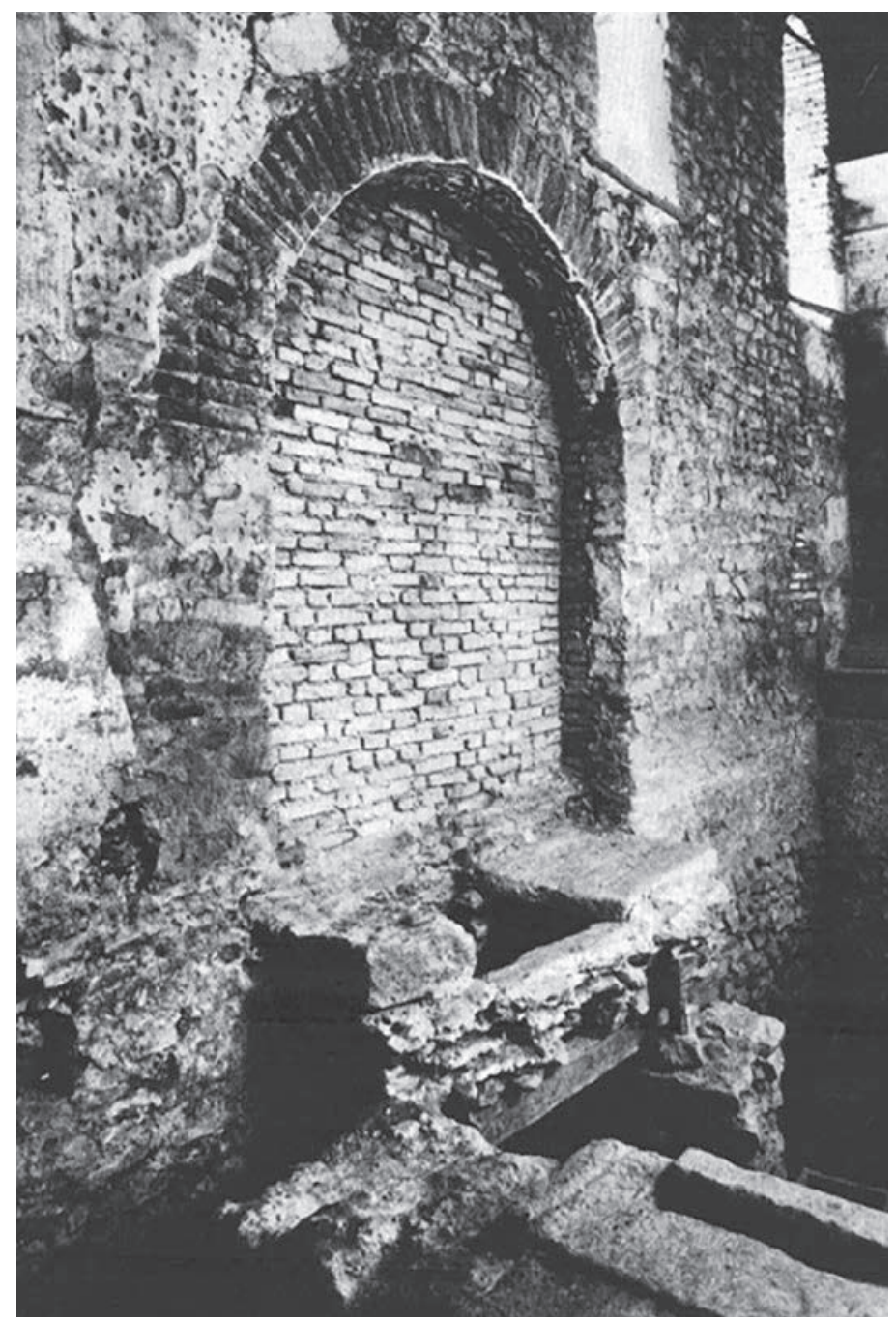

Fig. 6. Brescia, San Salvatore II. La tomba ad arcosolio dopo lo scavo (da G.P. BROGIOLO, 2014).

ticolare tipologia di manufatti, facendo leva sull'aspetto dimensionale degli stessi e ponendolo a confronto con i dati archeologici superstiti. La tomba ad arcosolio inserita nel muro perimetrale sud del San Salvatore II di Brescia ${ }^{30}$ non escluderebbe ad esempio, per diversi ordini di motivi, una certa coerenza con l'inserimento di una lastra funeraria a impianto orizzontale a doppia colonna, essendo la sua parete di fondo predisposta a contenere un manufatto di almeno $200 \mathrm{~cm}$ di larghezza ${ }^{31}$ (fig. 6).

\footnotetext{
${ }^{27}$ Sull'epigrafe di Cuniperga si veda A. SILVAGNI, Monumenta epigraphica christiana, op. cit. (n. 16), tab. II, ${ }^{\circ}$ 3; N. GRAY, The palaeography of latin inscriptions, op. cit. (n. 16), p. 76, n. 45; G. PANAZZA, Lapidi e sculture paleocristiane e pre-romaniche di Pavia, in Arte del primo millennio, Atti del II Convegno per lo studio dell'arte dell'alto medioevo (Università di Pavia, settembre 1950), Torino, 1953, p. 211-296, in part. p. 263-264, n. 75; A. PERONI, Musei d'Italia-Meraviglie d'Italia. Pavia, Musei Civici del Castello Visconteo, Bologna, 1975, p. 30, n. 130; A. PETRUCCI, Le scritture ultime, op. cit. (n. 16), p. 54; F. DE RUBEIS, La tradizione epigrafica longobarda nei ducati di Spoleto e Benevento, in I Longobardi dei ducati di Spoleto e Benevento, Atti del XVI Congresso internazionale di studi sull'alto medioevo (Spoleto, 20-23 ottobre 2002; Benevento, 24-27 ottobre 2002), Spoleto, 2003, p. 481-506, in part. p. 487; S. LOMARTIRE, Commacini e marmorarii. Temi e tecniche della scultura tra VII e VIII secolo nella Langobardia Maior, in I magistri commacini. Mito e realtà del medioevo lombardo, Atti del XIX Congresso internazionale di studio sull'alto medioevo (Varese-Como, 23-25 ottobre 2008), Spoleto, 2009, p. 151-209, in part. p. 198.

${ }_{28}$ Assegnata dalla Gray al 750 (N. GRAY, The palaeography of latin inscriprions, op. cit. [n. 16], p. 74-75, n. 43) e successivamente al 735-750 da Gaetano Panazza (G. PANAZZA, Lapidi e sculture paleocristiane, op. cit. [n. 27], p. 256-259, n. 66), l'epigrafe è attualmente custodita nei Musei Civici del Castello Visconteo di Pavia.

${ }^{29}$ Un numero complessivo di quattordici epigrafi, integre o mutile, reimpiegato sino al 1981 nelle murature di Casa Anelli e attualmente custodito presso i Musei Civici di Pavia, costituisce la preziosa testimonianza di una consistente parte della produzione epigrafica pavese della prima metà dell'VIII secolo. Appartengono a questo gruppo epitaffi più o meno lunghi aventi tra loro caratteristiche comuni. Sotto il profilo contenutistico, solo alcuni di questi possono fornire sommarie informazioni sul defunto, mentre per il resto è possibile muoversi nell'ambito delle ipotesi di carattere tecnico e paleografico. Questa raccolta epigrafica è stata pubblicata integralmente da Gaetano Panazza, per cui si veda G. PANAZZA, Lapidi e sculture paleocristiane, op. cit. (n. 27), numeri $67-73,75,79,82-86$.

$3^{30} \mathrm{Si}$ veda, da ultimo, G.P. BROGIOLO, Archeologia e architettura delle due chiese di San Salvatore, in Id., F. Morandini (a cura di), Dalla corte regia al monastero di San Salvatore-Santa Giulia di Brescia, Verona, 2014, p. 35-87, in part. p. 53.
}

${ }^{31}$ F. DE RUBEIS, Modelli impaginativi delle iscrizioni funerarie, op. cit. (n. 14), p. 61-62. 


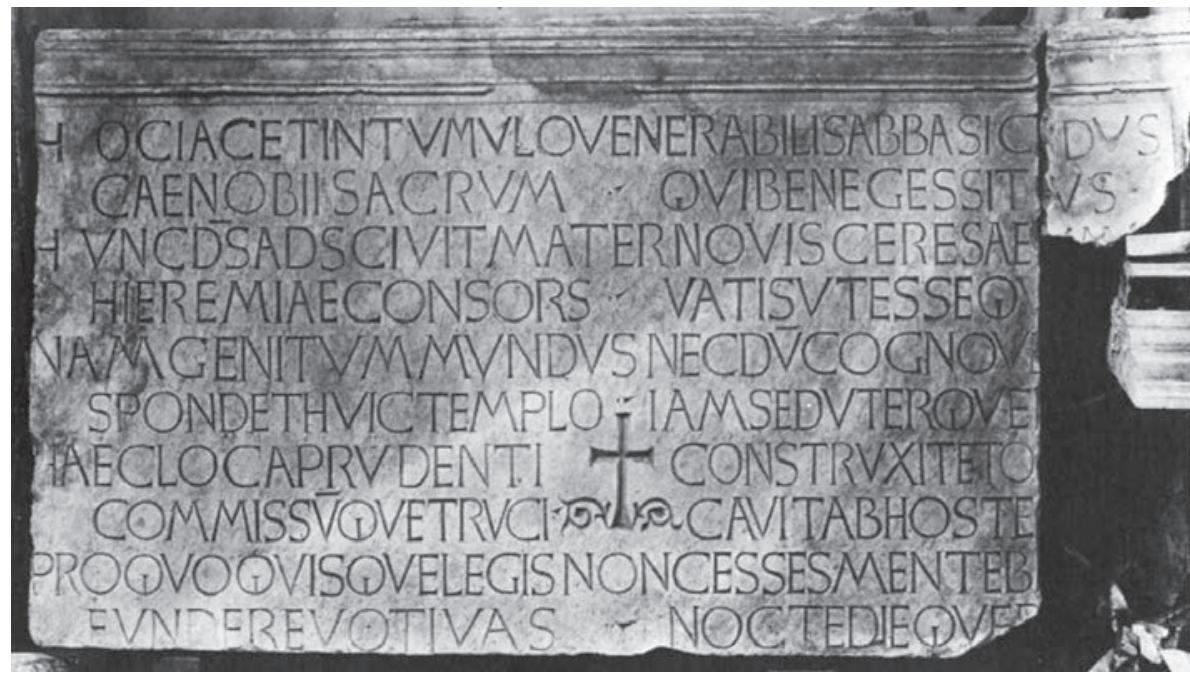

Fig. 7. Farfa, epigrafe funeraria dell'abate Sicardo († 842) (da C. McCLANDON, 1987). motivi iconografici. La necessità di rendere visibili i temi raffigurati ed enfatizzare così il luogo di sepoltura suggerirebbe una collocazione isolata dei sarcofagi a parete, per la quale pare assai suggestiva l'ipotesi che le lastre riportanti gli elogi di sovrani e badesse fossero posizionate nella parete, in corrispondenza del sepolcro e in posizione visivamente frontale rispetto all'osservatore. Si tratterebbe di una soluzione analoga a quella che si può offrire, senza alcuna distinzione, per le sepolture dei sovrani longobardi, il cui investimento dovette consistere, a partire dall'VIII secolo, non tanto (o non più) nella composizione del corredo, quanto piuttosto nella realizzazione di monumenti funerari quasi sempre in linea con la tradizione romano-imperiale e quindi composti

Sulla scorta di queste osservazioni, si potrebbe suggerire, tuttavia, anche una seconda soluzione a questa particolare tipologia di impianto. Ancora dal monastero bresciano provengono una serie di bellissimi frammenti di sarcofago di età romana, venuti alla luce durante gli scavi del Panazza e a seguito delle attività di sistemazione condotte nel 1998. Essi raffigurano le Amazzoni, le stagioni, le tre Grazie, Giona, elementi e temi che non trovano corrispondenze in quelli, certamente più modesti, provenienti da altre aree della città $^{32}$. Recentemente, Gian Pietro Brogiolo, riprendendo un discorso già affrontato in altra sede ${ }^{33}$, ha ricordato come in alcuni contesti di età longobarda non manchino esempi di sarcofagi di reimpiego destinati a contenere le spoglie di importanti personaggi, dalla tomba del cosiddetto duca Gisulfo a Cividale al caso di Spoleto, per non parlare del sarcofago intero che recava, secondo le fonti medievali, la breve iscrizione di Ansa ${ }^{34}$.

La presenza di manufatti di simile qualità potrebbe giustificare, dunque, la necessità di custodire in modo adeguato sacre reliquie $o$, in alternativa, la sepoltura di personaggi di altissimo livello, così come indicherebbe anche la scelta dei da un sarcofago e da un'iscrizione di carattere solenne ${ }^{35}$.

Così come per i carmina realizzati su marmo e destinati ai più alti esponenti dell'aristocrazia longobarda beneventana: improntate, con ogni probabilità, sui modelli italo-settentrionali dell'VIII secolo, le iscrizioni a doppia colonna dei principi Sicone $(†$ 832), Radelchi († 851), Radelgario († 854), della regina Caretruda († ante 875) e di suo figlio Orso ( $†$ ante 851 ), dovevano essere posizionate, infatti, a parete nel Paradisus della Cattedrale, un atrio porticato antistante l'ingresso alla basilica ${ }^{36}$. La versione ricavata dagli epitaffi stessi, trasmessi per tradizione diretta o indiretta, fornisce indicazioni locative che prediligono l'utilizzo di termini quali tumulum ${ }^{37}$ e monumentum ${ }^{38}$, restituendo in questo modo anche l'immagine di sepolture fuori terra, dotate di specifiche peculiarità monumentali. L'assenza, nell'atrio, di tracce che possano ricondurre a strutture di tipo monumentale ${ }^{39}$, quali ad esempio quelle inerenti alle tombe ad arcosolio, rafforza l'ipotesi che le grandi lastre funerarie ( $200 \times 100 \mathrm{~cm}$ in media) adornassero in origine le pareti su cui erano addossati, verosimilmente, dei sarcofagi indipendenti.

\footnotetext{
${ }^{32}$ Sugli elementi di reimpiego, compresi i frammenti di sarcofago, si veda F. MORANDINI, La presenza dell'antico nelle strutture del monastero, in G.P. Brogiolo, Ead. (a cura di), Dalla corte regia, op. cit. (n. 30), p. 341-383. Sui frammenti di sarcofago, in particolare le p. 341-345.

33 G.P. BROGIOLO, Desiderio e Ansa a Brescia: dalla fondazione del monastero al mito, in C. Bertelli, G.P. Brogiolo (a cura di), Il futuro dei Longobardi. L'Italia e la costruzione dell'Europa di Carlo Magno, Saggi, Milano, 200o, p. 143-155, in part. p. 153.

${ }^{34}$ G.P. BROGIOLO, Archeologia e architettura delle due chiese di San Salvatore, op. cit. (n. 30), p. 57. Su questo punto, bisognerà tenere in debito conto anche le osservazioni di Francesca Morandini, che reputano poco plausibile lo smantellamento di sepolture di pregio in un edificio a continuità di culto, per cui si veda F. MORANDINI, La presenza dell'antico nelle strutture del monastero, op. cit. (n. 32), p. 348. Secondo Giancarlo Andenna, in un saggio meno recente, la distruzione dei sarcofagi sarebbe avvenuta in maniera intenzionale, in segno di spregio a seguito della sconfitta di Desiderio, cfr. G. ANDENNA, La vita e il ruolo del monastero, in R. Stradiotti (a cura di), San Salvatore-Santa Giulia a Brescia, il monastero nella storia, Milano, 2001, p. 41-53.

${ }^{35}$ Le fonti di età longobarda svelano, talvolta, dettagli interessanti sulle pratiche funerarie, con particolare riferimento a personaggi illustri e ai sovrani. A seguito della sepoltura in Sant'Adriano, presso Santa Maria in Pertica a Pavia, le spoglie di Liutprando furono traslate, ad esempio, nella chiesa di San Pietro in Ciel d'Oro e in quella occasione fu eretto un monumento funerario composto da un sarcofago marmoreo sopraelevato su colonnine. Sul tema si veda P. MAJOCCHI, La morte del re. Rituali funerari regi e commemorazione dei sovrani nell'alto medioevo, in Storica, 49, 2011, p. 7-61.

${ }^{36}$ Su queste ipotesi cfr. C. LAMBERT, La produzione epigrafica nei secoli VIII e IX in Salerno e Benevento, in G. Roma (a cura di), I Longobardi del Sud, Roma, 2010, p. 291-322, in part. p. 296. La fonte più antica che documenta l'esistenza dell'atrio è un documento del 1136, mediante cui il papa Anacleto II concede in uso a Rossemanno, arcivescovo di Benevento, il paradiso del Duomo per le attività commerciali (tràdito in F. UGHELLI, Italia sacra sive de episcopis Italiae et insularum adiacentium, VIII, Venetiis, 1721, p. 109-110).

${ }^{37}$ Il termine è riportato all'interno dell'epigrafe di Radelgario. Edizione e traduzione integrale in C. LAMBERT, La produzione epigrafica nei secoli VIII e IX, op. cit. (n. 36), p. 312-313, n. 12.

${ }^{38}$ Così come negli epitaffi di Radelchi e Caretruda, per cui Ibidem, p. 311-312, n. 9-10.

39 Indagini archeologiche hanno interessato l'intera area della cattedrale, portando alla luce anche le strutture riguardanti l'antico paradisus. In quest'area lo scavo ha evidenziato la presenza di alcune sepolture allineate su di un unico fronte e destinate, forse, a personaggi in vista dell'aristocrazia beneventana. Per una breve panoramica sui risultati degli scavi si veda L. TOMAY, Benevento longobarda: dinamiche insediative e processi di trasformazione, in G. D'Henry, C. Lambert (a cura di), Il popolo dei Longobardi meridionali (570-1076). Testimonianze storiche e monumentali, Atti del convegno (Salerno, 28 giugno 2008), Salerno, 2009, p. 119-151, in part. p. 130-134.
} 
Quali che fossero le soluzioni adottate, gli spazi destinati alla memoria di badesse e regine in alcuni monasteri del Regnum - come i cenobi di Sant'Agata al Monte e Santa Maria Teodote a Pavia e il San Salvatore di Brescia - dovettero configurarsi come luoghi abbastanza ampi e aperti alla lettura pubblica, tali da conferire alle scritture esposte un'adeguata funzione commemorativa. Ne consegue che simili elementi avrebbero potuto convivere solo all'interno delle grandi abbaziali, ragion per cui le ipotesi che propongono l'impianto di sepolture di prestigio all'interno di questa particolare categoria di edifici risulterebbero ancora più concrete.

Situazione simile ma non del tutto identica potrebbe essere registrata per il periodo successivo. In età carolingia si assiste alla diffusione di un modello epigrafico di grandi dimensioni ea sviluppo orizzontale, connotato dalla presenza di un testo organizzato, questa volta, a piena pagina (cfr. fig. 2f). Rotazione dell'asse di scrittura e variazioni grafiche non costituiscono le uniche modifiche, poiché importanti differenze possono essere individuate nelle misure degli specchi e nello spessore delle lastre, fattore che contribuisce a rivedere le ipotesi circa l'ubicazione originaria dei manufatti.

Sulla base delle integrazioni tràdite nella Constructio, il testo inciso al di sopra della lastra di Sicardo ${ }^{40}$, abate di Farfa dall'830 all'842, è inserito all'interno di uno specchio delimitato da una cornice a doppio listello, aggettante rispetto all'area di scrittura di $2 \mathrm{~cm}$ circa. (fig. 7). L'incrocio dei dati materiali e di quelli ricavabili dalla fonte ha permesso di ricostruire un modello grafico dell'iscrizione calcolando la quantità delle lettere superstiti in ciascuna riga e restituendo così le misure di una lastra di più di $200 \mathrm{~cm}$ di larghezza e di $100 \mathrm{~cm}$ di altezza. Malgrado le evidenti analogie con la produzione italo-settentrionale per ciò che concerne le misure complessive della lastra, l'epigrafe di Sicardo si distingue tuttavia per lo spessore, raggiungendo lungo i margini i 10 cm circa, fattore che orienta verso l'attribuzione della lapide ad un contesto di notevole impatto visivo.

Forma e dimensioni di alcuni manufatti epigrafici hanno spinto abbastanza di recente ad individuare, in area francese e per l'età carolingia, l'utilizzo di epigrafi di tipo privilegiato, destinate ad occupare le superfici di importanti monumenti funebri ${ }^{41}$. Le parole contenute all'interno della Vita di Adalardo di Corbie, abate del cenobio francese dal 781 all'826, confermano il quadro così delineato, documentando la presenza, al centro della chiesa abbaziale, di un impianto monumentale destinato a contenere le spoglie dell'abate, così descritto: «sepulta sunt autem decenter membra carissimi senis in basilica beati Petri apostoli sub fastigio inter eiusdem medioximae quatuor ecclesiae centra, tectus polito lapide» ${ }^{42}$. La menzione del termine fastigium denota, in questo caso, l'occupazione di un'area vitale della chiesa da parte del monumento funebre, nonché l'elevata visibilità dell'iscrizione, «polito lapide: super quem octonis sculptum versibus musici carminis haustu digne legitur» ${ }^{43}$, rispetto all'intero spazio circostante.

Sulla scorta di queste considerazioni, le modifiche riguardanti gli spessori di alcuni manufatti destinati a commemorare i presbiteri e gli abati potrebbero aver fatto capo anche ad un qualche tipo di evoluzione delle strutture funerarie, avvenuta verosimilmente con la comparsa di sepolcri dotati di specifiche peculiarità. Benché sia attualmente difficile risalire alla forma di questi ultimi, per via dell'assenza di adeguati riferimenti, si potrà comunque avanzare l'ipotesi di un loro sviluppo anche in ambito monastico italiano. Al di là dell'epigrafe di Sicardo, in questa direzione sembrerebbe condurre, infatti, anche l'analisi di una seconda iscrizione, rinvenuta nel 1893 all'interno del duomo Vecchio di Brescia e dedicata al sacerdos Tafo di Leno, datata all'11 aprile $897^{44}$ (cfr. fig. 2f). L'appartenenza dell'oggetto ad un contesto di natura elitaria può essere ricavata dall'analisi del testo inci$\mathrm{so}^{45}$, per cui si può denotare l'utilizzo di formule complesse, quali spiritus astra petit, o, in alternativa, dal suo stesso aspetto dimensionale. L'integrazione delle lacune testuali in corrispondenza del margine destro dell'epigrafe permette di risalire approssimativamente alla larghezza originaria della lastra, di circa $140 \mathrm{~cm}$, benché l'aspetto più sorprendente della testimonianza vada rintracciato nei suoi $20 \mathrm{~cm}$ di spessore. Escludendo, per queste stesse caratteristiche, l'inserimento della lastra a pavimento o su di una superficie muraria, l'ipotesi di un suo innesto originario all'interno di un monumento funerario sembrerebbe essere la più plausibile.

Riduzione dello specchio di scrittura e incremento significativo degli spessori sembrano voler riflettere le esigenze dettate dalla diffusione di un modello funerario di tipo monumentale destinato, a quanto pare, ad una committenza selezionata. Gli unici confronti possono essere istituiti, infatti, con l'epigrafe dell'abate ignoto di Leno ${ }^{46} \mathrm{e}$, per ciò che concerne le dimensioni, con l'iscrizione dell'abate Magnus ${ }^{47}$. Naturalmente, la produzione di età carolingia non si esaurì con quegli esempi, come testimonia la persistenza, benché in minor misura, delle pratiche diffuse in età longobarda.

\section{ISCRIZIONI PARIETALI DI MEDIE E PICCOLE DIMENSIONI}

Qualora da manifestazioni di carattere elitario, o quanto meno riservato, si passi ad esaminare la produzione destina-

\footnotetext{
${ }_{40}$ Sulla lastra di Sicardo si veda la scheda di F. BETTI, Corpus della scultura altomedievale, XVII. La Diocesi di Sabina, Spoleto, 2005, p. $123-127$, n. 66.

${ }^{41}$ Cfr. C. TREFFORT, Mémoires carolingiennes, op. cit. (n. 10), p. 125-126.

${ }^{42} \mathrm{MGH}$, Scriptores Rerum Langobardicarum et Italicarum, op. cit. (n. 8), II, 532.

43 Ibidem.

${ }^{44}$ M. SANNAZARO, Le iscrizioni paleocristiane e altomedievali da Leno, op. cit. (n. 19), p. 93-95.

45 «qui fuerat mitis patiens humilisq(ue) sacer[dos] / ingenio pollens nobilitate vigens / vita nam semper Xpi(sti)i spem rite regebat / illius metam novimus esse bonam / tum proprio reddens animam de corpore pulchr[am] / corpus humo sepelit sp(iritu)s astra petit / vos rogo lectores qui carpitis acta Taphonis / in D(omi)no valeas dicite corde pio / anno Dominicae incarnationis DCCCXCVII ind(ictione) [...] /(tertio die) id(uum) aprilium feliciter migravit ad $\mathrm{Xp}(\mathrm{istu}) \mathrm{m}$.

${ }^{46}$ M. SANNAZARO, Le iscrizioni paleocristiane e altomedievali da Leno, op. cit. (n. 19), p. 350-352.

${ }^{47}$ Per l'iscrizione dell'abate Magnus di Leno si veda O. BANTI, Considerazioni a proposito di alcune epigrafi dei secoli VIII-IX conservate a Brescia, in Santa Giulia di Brescia. Archeologia, arte e storia di un monastero regio dai Longobardi al Barbarossa, Brescia, 1992, p. 163-177, in part. p. 172-173 (che data agli inizi del X secolo). Cfr., inoltre, F. DE RUBEIS, La produzione epigrafica priuma e dopo il 774, in S. Gasparri (a cura di), 774, ipotesi su una transizione, Atti del seminario di studio (Poggibonsi, 16-18 febbraio 2006), Turnhout, 2008, p. 403-422, in part. p. 421.
} 


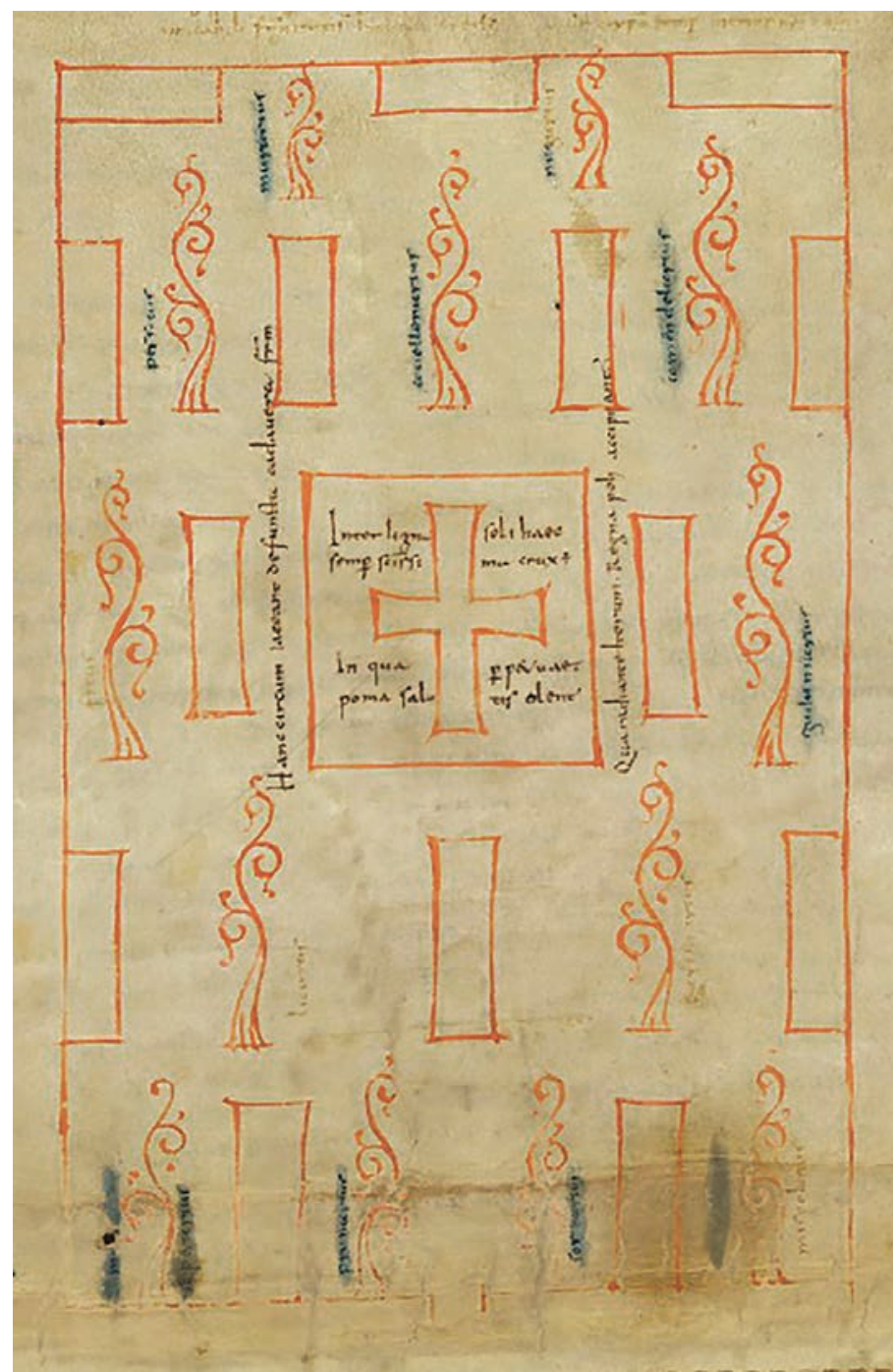

Fig. 8. Sankt Gallen Stiftsbibliothek. Ms. 1092, "Pianta di San Gallo", particolare del cimitero dei monaci (a. 820).

ta agli esponenti meno in vista della gerarchia cenobitica, il quadro che si profila è di gran lunga più articolato.

Che l'area cimiteriale destinata alla comunità monastica costituisse di per sé un corpo unico e ben distinto rispetto alle restanti zone del cenobio è confermato dalla celebre pianta di San Gallo realizzata tra l'816 e l'823 $3^{48}$. Benché si tratti di un progetto mai eseguito, il documento costituisce un modello ideale per lo studio dell'architettura monastica di età carolingia, facendo spesso da base anche alle indagini archeologiche. All'interno della pianta un nucleo isolato di sepolture, ubicato a est della chiesa abbaziale, è distribuito intorno ad una grande croce centrale, ai cui lati è possibile osservare la didascalia «hanc circum iaceant defuncta cadavera fratrum qua radiante iterum regna poli accipiant | inter ligna soli haec semper sanctissima crux». Nulla viene lasciato al caso, poiché l'unico elemento segnaletico sembra essere costituito dalla croce centrale e dal gruppo di alberi da frutto di cui il compilatore indica volutamente il nome (fig. 8 ). Nessun segno relativo a scritture che possano in qualche modo ricondurre alla sepoltura trova posto all'interno del progetto, come se il ricordo dei monaci fosse già sufficientemente tutelato dall'amenità del luogo.

Nel periodo che va a partire dalla fine dell'VIII secolo, il cenobio di San Vincenzo al Volturno, nel Molise interno, assiste alla comparsa di un primo gruppo epigrafico riservato ai monaci, composto da manufatti di forma quadrata, di dimensioni regolari che si aggirano in media intorno ai $20 / 25 \mathrm{~cm}$ per lato. Si tratta di epigrafi funerarie dalla struttura testuale piuttosto semplice ma dalle particolarità tecniche e grafiche di tipo avanzato. Procedendo nel campo delle testimonianze di cui è possibile ricostruire il profilo originario, il gruppo di esemplari assegnabili al monastero annovera al proprio interno anche epigrafi di forma rettangolare e quadrata dalle dimensioni maggiormente ridotte, mostrando in media l'utilizzo di un modulo di $20 \mathrm{~cm}$ di altezza e $15 \mathrm{~cm}$ di larghezza, con la comparsa anche di alcuni casi eccezionali.

L'esistenza di modelli epigrafici omogenei di forma quadrangolare o lievemente sviluppata in verticale può essere documentata, ad esempio, da un'epigrafe recentemente ricomposta. Lanalisi autoptica delle superfici lavorate ha permesso di stabilire contatti tra alcuni reperti messi in luce nell'anno $2007^{49}$ e altri frammenti provenienti dall'area del refettorio degli ospiti $i^{\circ}$, restituendo così una porzione consistente di un'iscrizione funeraria dedicata ad un monaco di nome Hermolus (fig. 9). L'apparato grafico, che ben si inserisce nello scenario vulturnense della prima metà del secolo IX ${ }^{51}$, è eseguito all'interno di uno specchio incassato, ricavato dalla lavorazione del coperchio di una probabile urna funeraria di età romana, di cui è ben visibile il motivo a nastri viminei intrecciati. L'aspetto maggiormente affascinante di questa testimonianza risiede, tuttavia, nella rilavorazione medievale dell'urna, poiché le modalità del suo riutilizzo non dipendono tanto dal suo aspetto estetico, quanto anche e soprattutto dalle dimensioni complessive. Considerando larghezza delle lettere e distanza media tra le stesse all'interno del testo, sarà possibile immaginare, infatti, a una lastra di forma quadrangolare di $20 \mathrm{~cm}$ per

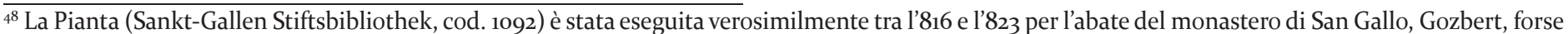
per ordine di Haito, guida della comunità di Reichenau. A partire dal primo studio analitico della pianta eseguito da Walter Horn e Ernest Born nel 1979 (W. HORN, E. BORN, The plan of St. Gall: a study of the architecture and economy of, and life in a paradigmatic carolingian monastery, 3 voll., BerkeleyLos Angeles-London, 1979) le indagini hanno visto un notevole incremento sino ai giorni nostri. Per un inquadramento generale del manufatto e una dettagliata rassegna bibliografica si veda la pagina www.stgallplan.org/en. Recenti spunti e revisioni in F. MARAZZI, Le città dei monaci. Storia degli spazi che avvicinano a Dio, Milano, 2015, p. 280-295.

${ }^{49}$ Campagna di scavo PZ 2007, RRNN 6166, 6168, 6169. Si veda, a tal proposito, D. FERRAIUOLO, I reperti epigrafici, in F. Marazzi, A. Luciano (a cura di), Iuxta flumen Vulturnum. Gli scavi lungo il fronte fluviale di San Vincenzo al Volturno, Modugno (Ba), 2015, p. 195-202, in part. p. 197-199.

${ }^{50}$ Campagna di scavo 1982. Sui frammenti, RRNN 1546, 1547, 1548, 1550, si veda J. MITCHELL, Late antique and early medieval carved inscriptions, op. cit. (n. 20), p. 53-54, n. 42 .

${ }^{51}$ La bibliografia riguardante lo sviluppo dell'epigrafia in ambito vulturnense è piuttosto nutrita. Per una panoramica generale sull'evoluzione delle forme grafiche, si vedano F. DE RUBEIS, La scrittura a San Vincenzo al Volturno, op. cit. (n. 2o); J. MITCHELL, Late antique and early medieval carved inscriptions, op. cit. (n. 20). Per gli ultimi aggiornamenti, cfr. D. FERRAIUOLO, Scrivere, impaginare, scolpire, op. cit. (n. 20), p. 79-10o; ID., Tra canone e innovazione. Lavorazione delle epigrafi nella Langobardia Minor (secoli VIII-X), Borgo San Lorenzo (Fi), 2013, in part. p. 49-57; F. DE RUBEIS, Il monastero di San Vincenzo al Volturno e le sue scritture, in H. Solin (a cura di), Le epigrafi della Valle di Comino, Atti del decimo convegno epigrafico cominese (Sora, $1^{\circ}$ giugno 2013), San Donato Val di Comino (Fr), 2014, p. 95-113.
} 


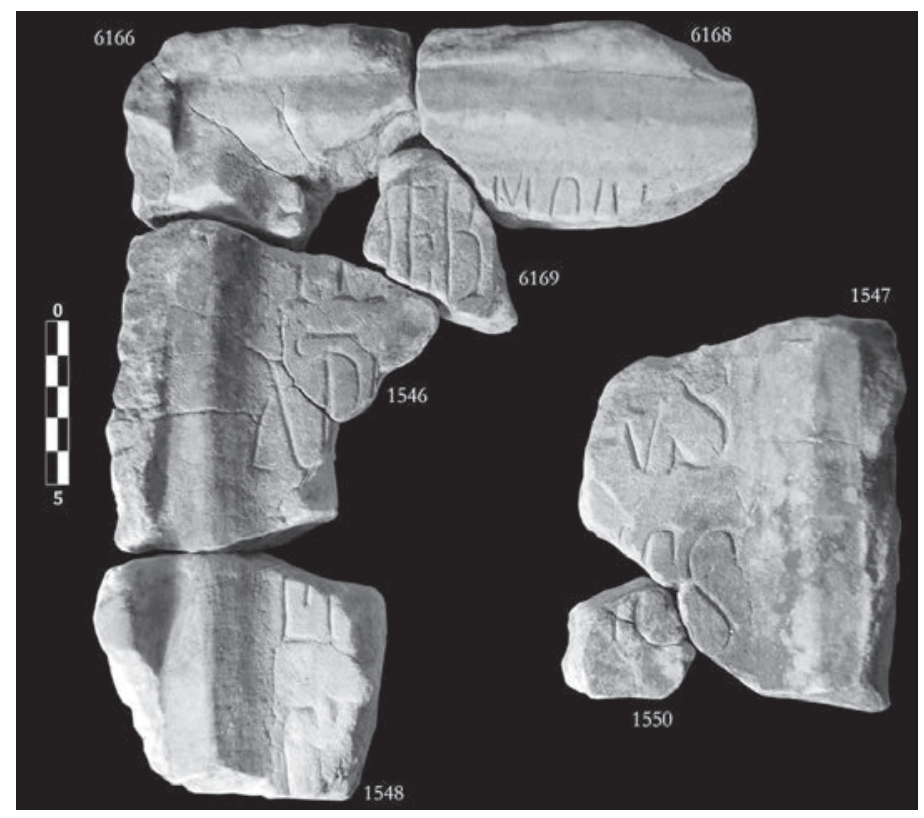

Fig. 9. San Vincenzo al Volturno. Frammenti dell'epigrafe funeraria di Hermolus (prima metà del IX secolo). lato, fattore che contribuisce ad alimentare l'ipotesi che le scelte del lapicida siano state indirizzate verso il riutilizzo di un oggetto avente dimensioni quanto più prossime a quelle delle lastre quadrangolari in uso a San Vincenzo al Volturno durante lo stesso periodo ( $25 \times 25 \mathrm{~cm}$ circa).

Nessuna differenza notevole, dunque, rispetto al quadro tracciato da un gruppo abbastanza nutrito di testimonianze dotate di analoghe particolarità estetiche e geometriche. Tenendo in considerazione i soli reperti integri o di cui è possibile ricostruire con certezza il profilo originario, appartengono a questo gruppo, ad esempio, anche le epigrafi dei monaci Ermecausus ${ }^{52}$ (fig. 10a), Maio ${ }^{53}$ (fig. 1ob), [...] $e^{2}{ }^{54}$ (fig. 10c), del magister (scriptorii?) Almo ${ }^{55}$ (fig. 1od), del presbiter Magnus ${ }^{56}$ (fig. 10e) e di un non meglio identificabile defunto ${ }^{57}$ (fig. 1of).

Epigrafi così organizzate - talvolta, come si è visto, di dimensioni molto ridotte - non riuscirebbero ad assolvere alle rispettive funzioni se ubicate in corrispondenza delle singole sepolture, mentre sarebbe più sensato un loro coinvolgimento all'interno di un contesto "comune" di tipo funerario. A tal proposito, non mancano gli esempi riconducibili a sepolture multiple, come indicherebbe, tra le

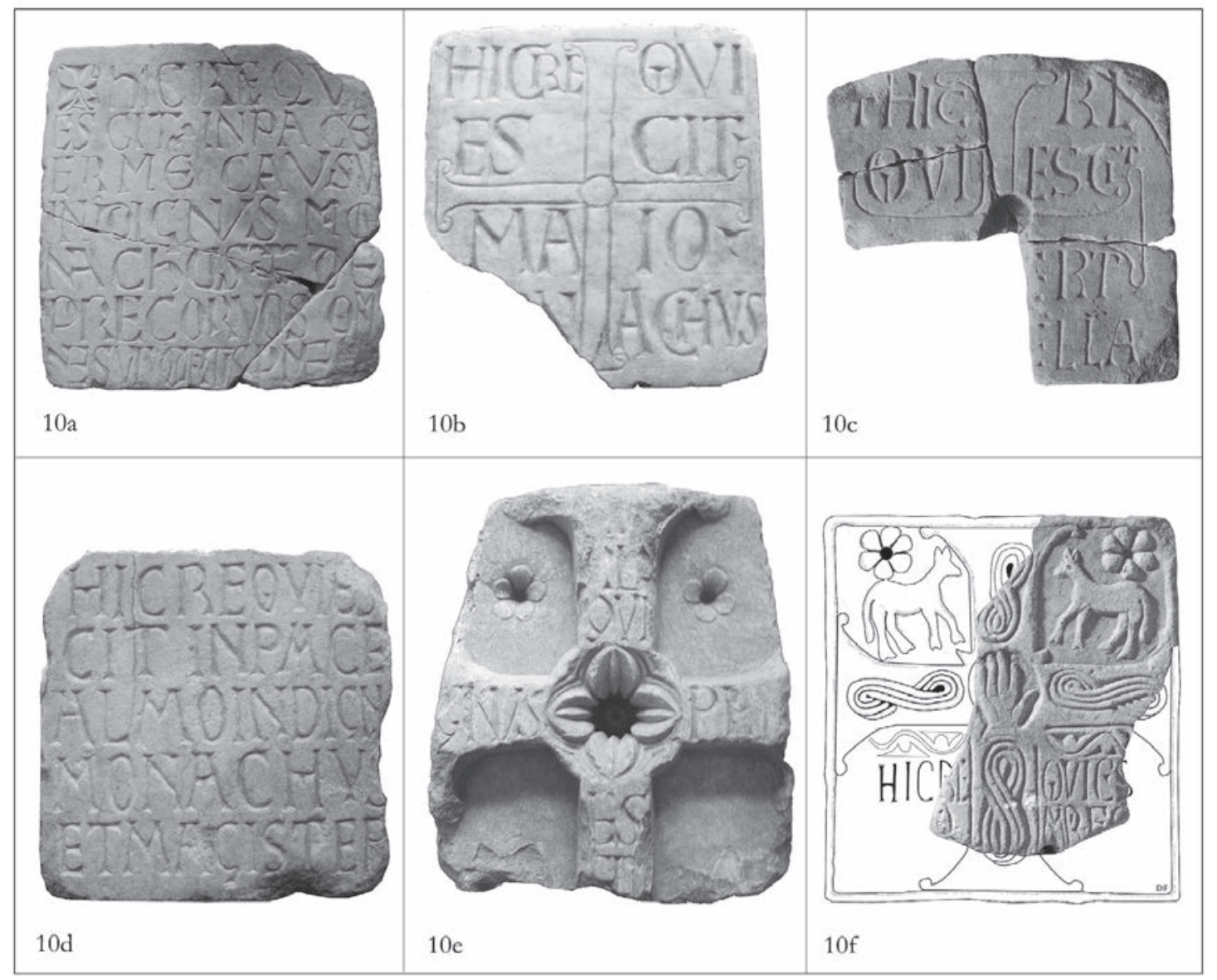

Fig. 10. Alcuni esempi di epigrafi di formato quadrato in uso a San Vincenzo al Volturno durante il IX secolo.

${ }_{52} \mathrm{RN} 3848$. L'iscrizione, attualmente al Museo di Venafro in mostra permanente, è tra le più note del vasto repertorio epigrafico vulturnense. Per la descrizione del pezzo, si veda F. DE RUBEIS, Iscrizione funeraria del monaco Ermecausus, in C. Bertelli, G.P. Brogiolo, Il futuro dei longobardi, op. cit. (n. 33), Catalogo, p. 143, n. 210; J. MITCHELL, Late antique and early medieval carved inscriptions, op. cit. (n. 20), p. 34 e 52-53, n. 41.

${ }_{53}$ Esemplare mutilo in marmo bianco, reimpiegato nella tessitura della pavimentazione in opus sectile della navata settentrionale della Basilica Maior, RN 5333 . Sulla contestualizzazione del manufatto si veda F. MARAZZI, La "Basilica Maior" di San Vincenzo al Volturno (scavi 2000-2007), Modugno (Ba), 2014 , p. 116.

${ }^{54}$ Manufatto disperso, per cui J. MITCHELL, Late antique and early medieval carved inscriptions, op. cit. (n. 20), p. 61, n. 6o, con relativo apparato bibliografico. ${ }^{55} \mathrm{RN} 5394$, epigrafe inedita.

${ }^{56}$ RN 4957. Sulle particolarità stilistiche del manufatto si veda L. CATALANO, La produzione scultorea medievale nell'abbazia di San Vincenzo al Volturno, Napoli, 2008 (Quaderni della Ricerca Scientifica UniSob, 13).

${ }^{57}$ Epigrafe dell'Agnus Dei, RN 1604. Sull'epigrafe si veda, da ultimo, J. MITCHELL, The small finds, in R. Hodges, S. Leppard, J. Mitchell (a cura di), San Vincenzo Maggiore and its workshops, Oxford, 2011, p. 195-333, in part. p. 302-304, con relativi rimandi bibliografici. L'analisi stilistico-formale del pezzo è in L. 


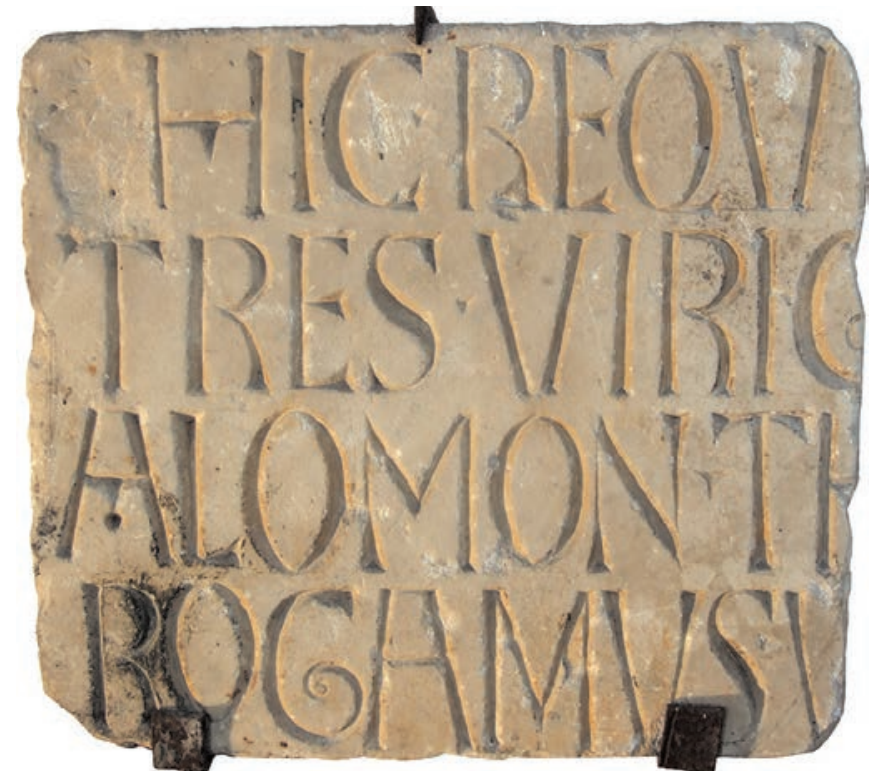

Fig. 11. Alvito (FR), Collezione Graziani. Epigrafe funeraria proveniente da San Vincenzo al Volturno (primo quarto del IX secolo, da G. DE BENEDITTIS, 2010).

altre, un'epigrafe poco nota e facente parte della collezione Graziani di Alvito ( $\mathrm{Fr}$ ), assegnata a San Vincenzo al Volturno sulla scorta del formulario e dell'apparato grafico ${ }^{58}$. In essa il riferimento ai tre defunti viri è contenuto in uno specchio rettangolare sensibilmente più ampio rispetto ai casi appena analizzati ${ }^{59}$, ma del tutto essenziale e privo di incorniciatura, a testimonianza di un suo impiego a parete (fig. 11).

Spunti interessanti giungono ancora dalla Francia merovingia e carolingia, dove si dispone di una discreta documentazione riguardante nello specifico la categoria delle epigrafi parietali. In questo senso, il confronto con un'epigrafe funeraria proveniente dalla chiesa di Sainte-Radegonde di Poitiers, luogo di inumazione delle monache del cenobio di Sainte-Croix, potrebbe risultare calzante, soprattutto per ciò che concerne il valore ideologico della testimonianza. Qui un piccolo mattone di pietra calcarea di $37,5 \mathrm{~cm}$ di larghezza e $15,7 \mathrm{~cm}$ di altezza (per uno spessore di $10 \mathrm{~cm}$ ) riporta il testo di un epitaffio destinato a tramandare la memoria della monaca Mumlenau, «defuncta cuius corpulus ic ( quiescit ${ }^{60}$. Forma e geometria del manufatto sembrano indicare che si tratti di un blocco da costruzione, ragion per cui si è potuto ipotizzare un suo impiego originario a parete, con la conseguente identificazione di un'iscrizione posta in un luogo chiuso e indirettamente collegata ad una sepoltura ivi custodita.

A San Vincenzo al Volturno, l'aspetto tecnico dei manufatti esclude che possa trattarsi di epigrafi poste a copertura del tumulo o, in posizione verticale, in corrispondenza della testata. L'ottimo stato di conservazione superficiale dei pezzi non sembra rivelare alcuna traccia di usura da calpestio, elemento questo che scarterebbe l'ipotesi di una ubicazione terragna delle epigrafi. A ulteriore sostegno di queste considerazioni, si osserva l'esistenza di iscrizioni eseguite a bassorilievo, per lo più connotate da una estrema varietà di temi raffigurati, il che avrebbe reso disagevole il passaggio in corrispondenza della loro superficie o nei loro pressi. Per citare un esempio, forse il più rappresentativo, la citata epigrafe del presbiter Magnus si distingue per la particolarità del messaggio iconografico riportato, mostrando l'impiego di fiori a quattro e cinque petali posti in corrispondenza dei quadranti incassati, mentre è lasciato alla croce a rilievo, aggettante di circa $3 \mathrm{~cm}$ rispetto allo specchio di corredo, il compito di accogliere sui bracci il breve testo inciso (cfr. fig. 10e).

L'ipotesi di una collocazione a parete delle lastre vulturnensi destinate ai monaci risulta, allora, più concreta, soprattutto se contestualizzata rispetto al quadro delineato dalle fonti. All'opera di Ato, abate del monastero dal 739 al 760 , si deve la costruzione di una chiesa di cui si ignora al momento l'ubicazione. A chiusura del libro terzo del Chronicon Vulturnense ${ }^{61}$, il monaco Giovanni afferma che «il luogo, all'interno della chiesa del beato Pietro, in cui erano stati deposti i corpi dei monaci, era frequentemente venerato da molti, poiché lì rifulgeva la luce divina»; aggiunge che «vi era uno stretto passaggio per accedervi, dal momento che la cripta funeraria [camera nel testo, n.d.t.] era stata costruita in modo da estendersi al di sotto di tutto lo spazio della chiesa, cosicché nella parte inferiore di essa si trovavano i corpi di costoro [i monaci, n.d.t.] eal di sopra quelli di molti abati, e ad entrambi venivano tributati gli uffici di una pia devozione ${ }^{62}$. Le parole del cronista danno adito ad alcune interessanti considerazioni sui luoghi destinati alla sepoltura della comunità, indicando come luogo principale proprio la chiesa di San Pietro che, stando al Chronicon, avrebbe svolto questa funzione per tutto il IX secolo ${ }^{63}$, almeno sino a quando «alcuni contadini ignoranti portarono colà il corpo

CATALANO, La produzione scultorea medievale nell'abbazia di San Vincenzo al Volturno, op. cit. (n. 56), p. 37-43, benché la presente ricerca si discosti dalle considerazioni che vedono nell'epigrafe una piccola parte di un più ampio manufatto artistico scolpito.

${ }^{8}$ Per l'analisi tecnica e paleografica dell'epigrafe, nonché l'edizione del testo, cfr. G. DE BENEDITTIS, Una [semi]nuova iscrizione altomedievale da San Vincenzo al Volturno e altro, in Considerazioni di storia e archeologia, 2010, p. 27-31.

59 Il reperto misura complessivamente 34 x 39,5 cm con uno spessore di 3,5 cm. Secondo le stime eseguite da Gianfranco De Benedittis, l'epigrafe sarebbe mutila di una riga scrittoria e di una porzione del margine destro. Sulla scorta di queste considerazioni, lo studioso propone la seguente edizione: «Hic requi[escunt] / tres viri G[uido S]/alomon Th[omas] / rogamus v[os ut] / [orate pro nos]» (Ibidem, p. 30). Tuttavia, come conferma l'analisi delle formule documentabili in ambito vulturnense - tra cui, a titolo esemplificativo, quella in uso per l'epigrafe di Ermecausus - suggerisce l'utilizzo della forma al congiuntivo, ut oretis pro nos.

${ }^{60}$ «In anno XX[...] regnante / K(a)rolo rege Francorum VIIII kal(endas) Iun(ia)s / sic fuid Mumlenau D(e)o d(e)vota / defu(n)ct(a) cuius [cor]p[u]sculus ic / [requ]iescit requies[cat] [anima ei]us in p[ace]». Per il manufatto, Poitiers (86) fine del secolo VIII, si veda C. TREFFORT, Mémoires carolingiennes, op. cit. (n. 10), p. 128-129 e relativi rimandi bibliografici.

${ }^{61}$ Chronicon Vulturnense del monaco Giovanni (= CV), ed. V. Federici, 3 voll., Roma, 1925-1938 (Fonti per la storia d'Italia, 58-6o).

${ }^{62}$ Chronicon Vulturnense del monaco Giovanni, scritto intorno all'anno 1130, ed. M. Oldoni (trad. L. De Luca Roberti), Isernia, 2010, p. 218. Testo originale in CV I, 375: «cum enim locus ille, in quo beatorum corpora monachorum in ecclesia Beati Petri posita fuerant, frequenter veneraretur a multis, maxime quia divina ibis epe micuerint luminaria, essetque patulus ibidem introeundi aditus, siquidem per tocius ecclesie corpus magni edificii camera ita constructa habebatur, ut horum infra eiusdem camere spacio corporibus repositis, desuper vero multorum abbatum, <ac $>$ utrisque pie devocionis exhiberentur officia». ${ }^{63}$ F. MARAZZI, Le città dei monaci, op. cit. (n. 48), p. 173-174. Non è possibile stabilire, allo stato attuale delle ricerche, l'ubicazione esatta del cimitero monastico di IX secolo, in base ai risultati delle indagini archeologiche. Considerando la tipologia delle croci dipinte all'interno di alcune sepolture e i rapporti stratigrafici delle strutture dell'atrio della Basilica Marior, la presente ricerca si discosta dal giudizio espresso in R. Hodges, S. Leppard, J. Mitchell (a cura di), San Vincenzo al Volturno and its workshops, op. cit. (n. 57), p. 98-104, secondo cui il sepolcreto ivi rinvenuto sarebbe da assegnare al IX secolo. 
d'una donna defunta; da allora l'ingresso di quel luogo è rimasto chiuso, in modo che quelli o altri, o altri ancora che possono portare altro, mai possano trovarne l'accesso ${ }^{64}$.

Le indicazioni riportate nella fonte pongono l'accento sull'ampiezza dell'edificio, specificando che lo spazio destinato ai monaci, corrispondente alla cripta, fosse ampio quanto l'edificio soprastante e quindi sufficientemente spazioso da ospitare, al di sotto del pavimento, le tombe di tutti i monaci appartenenti alla comunità. In questo contesto, la segnalazione delle singole sepolture sarebbe risultata impossibile, se non attraverso l'utilizzo di messaggi stringati, naturalmente collocati a parete a breve distanza l'uno dall'altro. Si potrà individuare destinazione d'uso $\mathrm{e}$ valore di queste testimonianze, allora, a partire dal formulario, facendo leva sulla presenza dominante dell'indicazione locativa hic requiescit. In questo caso, la menzione non intende designare, quasi certamente, una topografia precisa del sepolcro, quanto invece uno spazio delimitato probabilmente da un recinto, da muri o inerente l'interno di una chiesa o di una cripta. Si tratterebbe, forse, di quel tipo di rapporto dell'epigrafe con il corpo del defunto che Armando Petrucci ha definito «in absentia» e che indica l'insieme degli scritti lontani dal cadavere ma ugualmente rivolti a tramandarne la memoria ${ }^{65}$. Procedendo nell'ambito di queste ipotesi, e tenendo in debito conto la consistenza del corpus epigrafico vulturnense, si potrà ottenere, dunque, l'immagine di un grande edificio a due piani pullulante di scritture, individuando per il piano superiore l'innesto di lastre tombali di grandi dimensioni prevalentemente a pavimento e per quello inferiore qualcosa di molto vicino ad un grande mosaico parietale, composto di vere e proprie "tessere epigrafiche" (fig. 12).

Per concludere, non si potrà tuttavia considerare inconsueto il caso vulturnense, per via dell'esistenza di riferimenti specifici che attestino l'utilizzo di chiese funerarie a due piani già a partire dall'età paleocristiana e per le quali si potrebbe ipotizzare l'utilizzo delle medesime pratiche di carattere

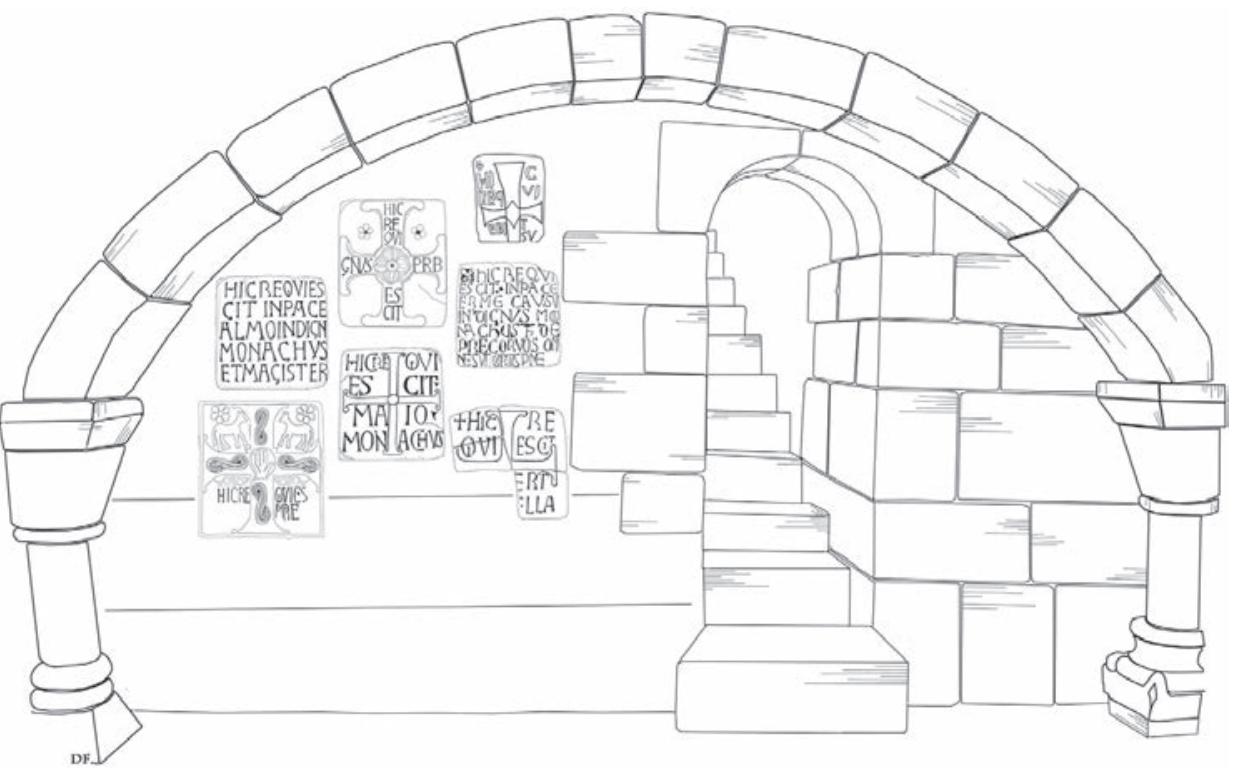

Fig. 12. Restituzione ideale della collocazione delle epigrafi vulturnensi di medie e piccole dimensioni (IX secolo).

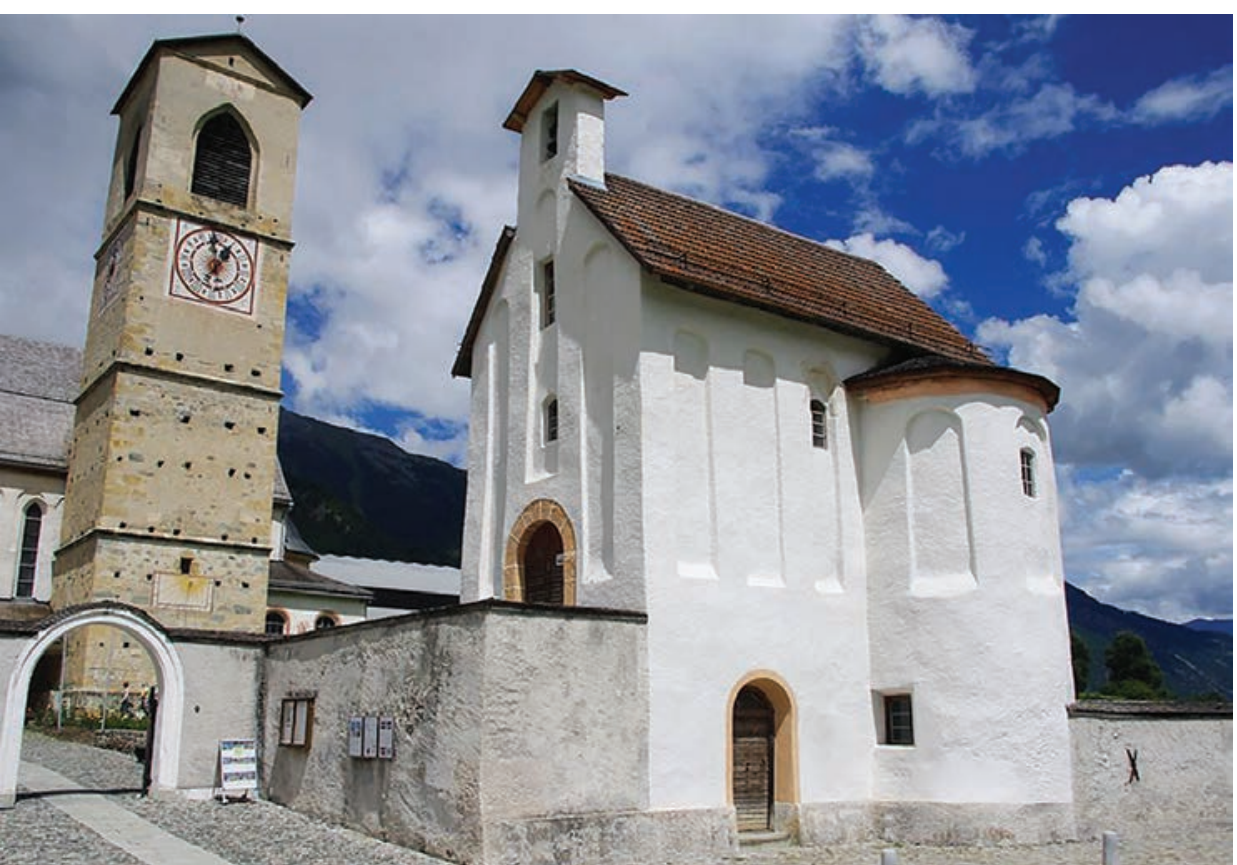

Fig. 13. Monastero di San Giovanni di Müstair, veduta della cappella della Santa Croce.

epigrafico ${ }^{66}$. Una versione superstite di questo modello architettonico potrebbe essere, ad esempio, quella offerta dalla cappella della Santa Croce annessa al monastero di San Giovanni di Müstair in Svizzera, che si può far risalire alla fine dell'VIII secolo sulla base delle indagini archeologiche svolte a partire dall'anno $1994^{67}$ (fig. 13). La cappella, a navata unica e terminazione triconca, si presenta ripartita in due piani, di cui quello inferiore impiegato, sin dal momento del

Si rimanda, per le ipotesi che datano il primo impianto dell'atrio e delle relative sepolture all'età dell'abate Giovanni IV (998-1007), alle considerazioni riportate in F. MARAZZI, La "Basilica Maior" di San Vincenzo al Volturno, op. cit. (n. 53), p. 147-232.

${ }^{64}$ «Quidam insipientes rustici de vicinis castellis, cuiusdam defuncte femine ibidem corpus intulerunt, atque ex tunc ita loci ipsius aditus clauditur, ut alterius vel quo ipsi introire, vel alios inferre possent, minime repperirent» (CV I, 375).

${ }_{65}$ A. PETRUCCI, Le scritture ultime, op. cit. (n. 16), p. XVIII.

${ }^{66} \mathrm{~A}$ tal proposito, costituirebbero validi modelli di riferimento i casi delle chiese funerarie di Saint-Laurent di Grenoble del IV-V secolo (R. COLARDELLE, Grenoble aux premiers temps chrétiens: Saint-Laurent et ses nécropoles, Paris, 1986) e di Saint-Victor di Marsiglia del X-XI secolo [P. AMARGIER, Un âge d'or du monachisme: Saint-Victor de Marseille (990-1090), Marseille, 1990; M. LAUWERS, Memoires des origins et ideologies monastiques. Saint Pierre des Fosses et Saint Victor de Marseille au XIe siècle, in Mélanges de l'École française de Rome, Moyen Âge, 115, 2003, p. 155-180), per le quali si può constatare l'uso, a scopo funerario, di ampie cripte sotterrane estese talvolta per una superficie pari a quella dell'edificio soprastante. 


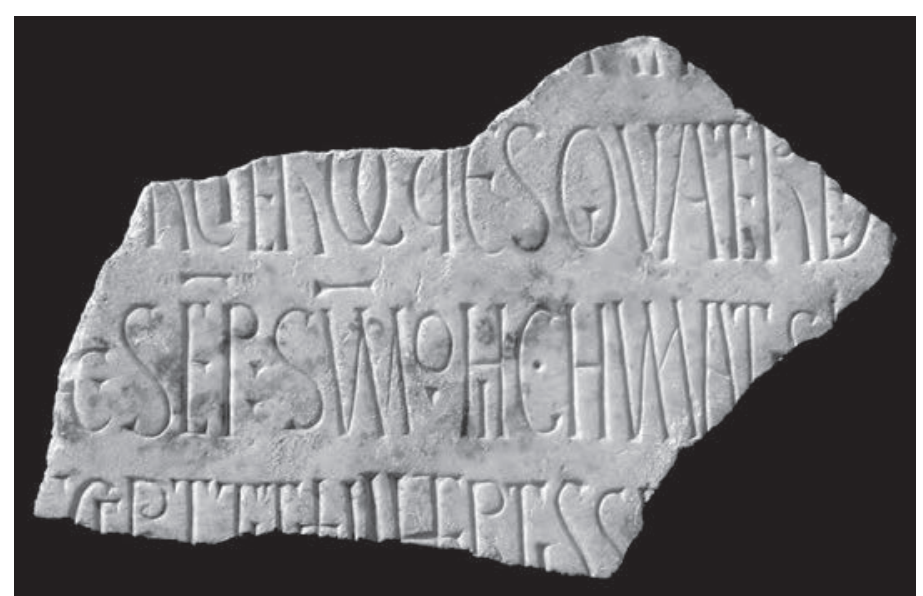

suo concepimento, come spazio riservato a cripta e luogo di sepoltura. Anche a Müstair il dato archeologico indicherebbe un uso diffuso delle lastre epigrafiche parietali, sebbene i rinvenimenti, in questo senso, siano molto meno numerosi rispetto al quadro nutritissimo di testimonianze vulturnen$\mathrm{si}^{68}$ (fig. 14). In questo panorama, quello di San Vincenzo al Volturno sembra porsi, ad ogni modo, come caso singolare per ciò che concerne la destinazione d'uso della cripta, poiché in linea di massima le ricerche indicano un utilizzo degli ambienti sotterranei come luogo di sepoltura per le personalità di alto rango, così come sembra potersi documentare per il caso svizzero cui si è appena dato accenno.

Fig. 14. Monastero di San Giovanni di Müstair, frammento di iscrizione funeraria (IX secolo, da F. DE RUBEIS, 2013).

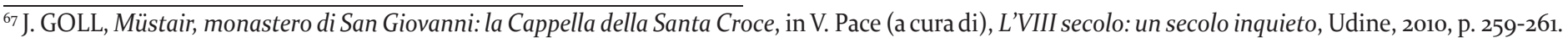
${ }^{68}$ Nell'anno 1997 è stato pubblicato il V volume del Corpus inscriptionum medii aevi Helvetiae a cura di Marina Bernasconi Reusser, contenente al proprio interno tutti i manufatti epigrafici rivenuti sino a quel momento all'interno del monastero di San Giovanni di Müstair, inquadrabili in un arco cronologico compreso tra l'VIII e il XVII secolo. Per ciò che concerne il materiale lapideo, il corpus CIMAH V contava solo 5 esemplari schedati, mentre i recenti aggiornamenti (F. DE RUBEIS, Il corpus epigrafico dell'abbazia di San Giovanni di Müstair, in H.R. Sennhauser (a cura di), Wandel und Konstanz zwischen Bodensee und Lombardei zur Zeit Karls des Grossen. Kloster St. Johann in Müstair und Churrätien, Acta (Tagung, 13-16 juni 2012), Zürich, 2013, p. 57-66) fanno salire a 45 il numero di testimonianze incise su marmo. Sulle scritture esposte a Müstair, con interessanti riferimenti anche al panorama epigrafico di Disentis, si veda anche M. BERNASCONI REUSSER, Le scritture esposte. Riflessioni sull'uso delle iscrizioni a Müstair e Disentis nell'alto medioevo, in H. Eisenhut, K. Fuchs, M. Hannes Graf, H. Steiner (eds.), Schrift, schriftgebrauch und textsorten im frühmittelalterlichen Chürratien, Vorträge des internationalen Kolloquiums (Chur Rätischen Museum, 18-20 mai 2006), Basel, 2008, p. 78-108. 